\title{
Mathematical Analysis and Numerical Simulations for a System Modeling Acid-Mediated Tumor Cell Invasion
}

\author{
Christian Märkl, ${ }^{1}$ Gülnihal Meral, ${ }^{2}$ and Christina Surulescu ${ }^{3}$ \\ ${ }^{1}$ Institut für Numerische und Angewandte Mathematik, Universität Stuttgart, Pfaffenwaldring 57, 70569 Stuttgart, Germany \\ ${ }^{2}$ Department of Mathematics, Faculty of Arts and Sciences, Bülent Ecevit University, 67100 Zonguldak, Turkey \\ ${ }^{3}$ Technische Universität Kaiserslautern, Felix Klein Zentrum für Mathematik, Paul Ehrlich Strasse, 67663 Kaiserslautern, Germany
}

Correspondence should be addressed to Christina Surulescu; surulescu@mathematik.uni-kl.de

Received 20 September 2012; Revised 26 December 2012; Accepted 3 January 2013

Academic Editor: Liancheng Wang

Copyright (c) 2013 Christian Märkl et al. This is an open access article distributed under the Creative Commons Attribution License, which permits unrestricted use, distribution, and reproduction in any medium, provided the original work is properly cited.

\begin{abstract}
This work is concerned with the mathematical analysis of a model proposed by Gatenby and Gawlinski (1996) in order to support the hypothesis that tumor-induced alteration of microenvironmental $\mathrm{pH}$ may provide a simple but comprehensive mechanism to explain cancer invasion. We give an intuitive proof for the existence of a solution under general initial conditions upon using an iterative approach. Numerical simulations are also performed, which endorse the predictions of the model when compared with experimentally observed qualitative facts.
\end{abstract}

\section{Introduction}

Despite major progress in medicine and science there still are incurable diseases which can threaten human lives. Cancer is among the most severe ones and it manifests itself as an uncontrolled growth of cells which are produced by the organism subsequently to mutations. Cancer cells migrate through the surrounding tissue and degrade it on their way toward blood vessels and distal organs where they initiate and develop further tumors, a process known as metastasis.

In the last decades various classes of models have been proposed aiming to provide a quantitative description of tumor growth. They range from the microscopic level of intracellular signaling pathways conditioning the growth of neoplastic tissue by stimulation or inhibition of apoptosis (e.g., by the influence of tumor necrosis factors [1]) or tumor cell motility, for example, by restructuring the cytoskeleton or by producing matrix degrading enzymes [2], through the level of cell-cell or cell-tissue interactions and up to the macroscopic level characterizing the behavior of the entire cell population. Multiscale settings like those in $[3,4]$ involve several of these scales and offer a systemic approach to the modeling process.

When ignoring the setups relying on mechanical force balance and/or on the theory of mixtures, in the study of tumor invasion and metastasis one can distinguish between the so-called kinetic approach and the direct modeling at the macroscopic level. In the former a mesoscopic model is considered, consisting of an integro-partial differential equation for the evolution of the cell density, possibly coupled with integro-differential and/or reaction-diffusion equations for the fibre density of the extracellular matrix (ECM) and the chemotactic signal (see, e.g., [5] and the references therein $[3,4])$. Then with an appropriate scaling the macroscopic limit is deduced, usually leading to a Keller-Segel-type model or some hyperbolic systems; see, for example, [6]. The macroscopic approach involves the largest class of existing models and directly accounts for processes at the level of cell populations, leading to systems of reaction-diffusion (transport) equations like, for example, in $[7,8]$.

The role of tumor microenvironment in determining cancer malignancy has been put in evidence in several references; see, for example, $[9,10]$. For instance hypoxia and acidity are factors that can trigger the progression from benign to malignant growth. In order to survive in the unfavourable environment they create, cancer cells upregulate certain proton extrusion mechanisms [11], the consequence of which is that the extracellular tumor environment has an acidic $\mathrm{pH}$, which boosts apoptosis of normal cells and thus allows the neoplastic tissue to extend in the space becoming available. 
Hence the $\mathrm{pH}$ level directly influences the metastatic potential of tumor cells [12, 13]. These facts led Gatenby and Gawlinski $[8,14]$ to propose a model for the acid-mediated tumor invasion, which describes the interaction between the density of normal cells, tumor cells, and the concentration of $\mathrm{H}^{+}$protons produced by the latter via reaction-diffusion equations. Starting from this model, travelling waves have been used to explain the aggressive action of cancer cells on their surroundings [15]. Further settings issued from Gatenby and Gawlinski's model involve nutrient dynamics influenced by both vascular and avascular growth of multicellular tumor spheroids $[16,17]$ assuming rotational symmetry and investigating existence and qualitative properties of the solutions.

In this work we reconsider the model in [8], whereby also explicitly allowing for crowding effects (due to competition with cancer cells) in the growth of normal cells. (This can be also done for the growth term in the tumor cell equation; however it does not change the analysis nor the qualitative behavior of the solutions to the system. Moreover, its biological motivation is not strong enough, since the competition between the two cell types does not really affect the growth, but rather the invasion of the neoplastic tissue: the acidity increase in the peritumoral environment is a byproduct of the enhanced glycolysis of cancer cells and not produced with the purpose of killing the normal cells and eluding concurrence.) For this setting we perform mathematical analysis and numerical simulations in order to verify the model predictions with respect to experimentally observed qualitative facts. In order to prove the existence of a unique (weak) solution we propose an intuitive method relying on an iterative procedure which has also been applied in $[18,19]$ in a different context (one of the supplementary difficulties here is the diffusion coefficient being nonconstant, but depending on the solution itself) and allows to avoid the use of operator semigroups.

\section{Problem Setting}

The model by Gatenby and Gawlinski [8] describes the evolution of normal and tumor cell density, respectively, in a domain where these cell types interact on the basis of $\mathrm{pH}$ value modifications. The mathematical description of these processes is ensured by the following system of reactiondiffusion equations for the normal cell density $N(t, \mathbf{x})$, the tumor cell density $K(t, \mathbf{x})$ (both in cells $/ \mathrm{cm}^{3}$ ), and the concentration of excessive $\mathrm{H}^{+}(t, \mathbf{x})$ ion concentration (in $\mathrm{Mol})$ :

$$
\begin{gathered}
\frac{\partial N}{\partial t}=w_{N} N\left(1-\frac{N}{K_{N}}-\theta \frac{K}{K_{K}}\right)-d_{N} H N \quad \text { in }(0, T) \times \Omega, \\
\frac{\partial K}{\partial t}=w_{K} K\left(1-\frac{K}{K_{K}}\right)+\nabla \cdot\left(D_{K}\left(1-\frac{N}{K_{N}}\right) \nabla K\right) \\
\text { in }(0, T) \times \Omega \\
\frac{\partial H}{\partial t}=w_{H} K-d_{H} H+D_{H} \Delta H \quad \text { in }(0, T) \times \Omega
\end{gathered}
$$

where $\theta \leq 1 / 2$ denotes the strength parameter for the competition between normal cells and neoplastic tissue. Thereby, $\Omega \subset \mathbb{R}^{n}(n=1,2,3)$ is a regular-enough and bounded domain and only microscopically small processes are considered at the interface between tumor and healthy tissue. Observe that the diffusion coefficient of the cancer cells depends on the normal cell density: when the healthy tissue is at its carrying capacity the neoplastic tissue cannot diffuse; thus the tumor is confined. It can only spread if the surrounding normal tissue is diminished from its carrying capacity and this is assumed to happen due to lowering the $\mathrm{pH}$ level upon secretion of $\mathrm{H}^{+}$protons by cancer cells.

The constants $w_{N}$ and $w_{K}$ are given in $1 / \mathrm{s}$ and represent the growth rates, and the constants $K_{N}$ and $K_{K}$ are expressed in cells $/ \mathrm{cm}^{3}$ and provide the carrying capacities of the normal and tumor cells, respectively. The death rate $d_{N}$ of the normal cells is measured in $1 /(\mathrm{Mol} \cdot \mathrm{s})$, and the diffusion coefficient of tumor cells in the absence of normal cells is given in $\mathrm{cm}^{2} / \mathrm{s}$. The production rate $w_{H}$ of $\mathrm{H}^{+}$protons is expressed in $\mathrm{Mol} \cdot \mathrm{cm}^{3} /$ (cells $\cdot \mathrm{s}$ ), the uptake rate $d_{H}$ (due for instance to proton buffering (see, e.g., [20] and the references therein) and/or various ion exchangers between intracellular and extracellular domains (see e.g. [21])) is measured in $1 / \mathrm{s}$, and their diffusion coefficient of $D_{H}$ in $\mathrm{cm}^{2} / \mathrm{s}$.

We also assume that there is no exchange of cells and $\mathrm{H}^{+}$protons through the boundary of the considered domain; thus

$$
\frac{\partial K}{\partial \mathbf{n}}=\frac{\partial H}{\partial \mathbf{n}}=0 \quad \text { in }(0, T) \times \partial \Omega,
$$

where $\mathbf{n}$ denotes the outer unit normal vector to $\partial \Omega$.

The initial conditions are given by

$$
\begin{gathered}
K(0, \mathbf{x})=K_{0}(\mathbf{x}), \quad N(0, \mathbf{x})=N_{0}(\mathbf{x}), \\
H(0, \mathbf{x})=H_{0}(\mathbf{x}) \quad \text { in } \Omega .
\end{gathered}
$$

Thereby $K_{0}(\mathbf{x}), N_{0}(\mathbf{x})$, and $H_{0}(\mathbf{x})$ are strictly positive functions, which satisfy the no-flux condition:

$$
\frac{\partial K_{0}}{\partial \mathbf{n}}=\frac{\partial H_{0}}{\partial \mathbf{n}}=0 \quad \text { on } \partial \Omega .
$$

In order to render the system (1) dimensionless we use the following transformations:

$$
\begin{gathered}
\widetilde{N}=\frac{N}{K_{N}}, \quad \widetilde{K}=\frac{K}{K_{K}}, \quad \widetilde{H}=H \cdot \frac{d_{H}}{w_{H} K_{K}}, \\
\widetilde{t}=w_{N} \cdot t, \quad \widetilde{\mathbf{x}}=\sqrt{\frac{w_{N}}{D_{H}}} \cdot \mathbf{x}
\end{gathered}
$$

along with the notations

$$
\begin{gathered}
\delta_{N}=\frac{d_{N} w_{H} K_{K}}{d_{H} w_{N}}, \quad \rho_{K}=\frac{w_{K}}{w_{N}}, \\
\Delta_{K}=\frac{D_{K}}{D_{H}}, \quad \delta_{H}=\frac{d_{H}}{w_{N}} .
\end{gathered}
$$


We obtain the system

$$
\begin{gathered}
\frac{\partial N}{\partial t}=N(1-N-\theta K)-\delta_{N} H N \\
\frac{\partial K}{\partial t}=\rho_{K} K(1-K)+\nabla \cdot\left(\Delta_{K}(1-N) \nabla K\right), \\
\frac{\partial H}{\partial t}=\delta_{H} K-\delta_{H} H+\Delta H,
\end{gathered}
$$

where for simplicity the tilde notations have been ignored. The stability analysis of this system (with $\theta=0$ ) has been performed in [8], leading to biologically significant predictions.

\section{Existence and Uniqueness of Solutions}

In this section we provide a natural proof for the existence and uniqueness of a weak solution to the system (1), with initial data (3) and boundary conditions (2). We make use of an iterative procedure instead of the classical approach via semigroup theory; this is more intuitive and allows for a separate treatment of the three equations in each step.

Consider the function spaces

$$
\begin{gathered}
X:=L^{\infty}\left(0, T ; H^{1}(\Omega)\right), \\
Y:=\left\{u \in L^{2}\left(0, T ; H^{2}(\Omega)\right): u_{t} \in L^{2}\left(0, T ; L^{2}(\Omega)\right)\right\}, \\
Z:=L^{\infty}\left(0, T ; L^{2}(\Omega)\right) .
\end{gathered}
$$

Definition 1. A weak solution of (1) with boundary conditions (2) and initial data (3) is a triple $(H, N, K)$ of functions in $X \times$ $Y \times Z$, such that for all $\phi \in H^{1}(\Omega)$ a.e. in $[0, T]$ the following three equations are satisfied:

$$
\begin{aligned}
& \int_{\Omega} w_{H} K \phi d \mathbf{x} \\
& =\int_{\Omega} H_{t} \phi d \mathbf{x}+\int_{\Omega} D_{H} \nabla H \nabla \phi d \mathbf{x}+\int_{\Omega} d_{H} H \phi d \mathbf{x}, \\
& \int_{\Omega} w_{N} N\left(1-\frac{N}{K_{N}}-\theta \frac{K}{K_{K}}\right) \phi d \mathbf{x} \\
& =\int_{\Omega} N_{t} \phi d \mathbf{x}+\int_{\Omega} N d_{N} H \phi d \mathbf{x}, \\
& \int_{\Omega} w_{K} K\left(1-\frac{K}{K_{K}}\right) \phi d \mathbf{x} \\
& =\int_{\Omega} K_{t} \phi d \mathbf{x}+\int_{\Omega} D_{K}\left(1-\frac{N}{K_{N}}\right) \nabla K \nabla \phi d \mathbf{x} .
\end{aligned}
$$

Theorem 2. There exists $T>0$, such that the system (1) with initial data (3) and boundary conditions (2) satisfying

$$
\begin{gathered}
H_{0} \in H^{1}(\Omega) \cap C(\Omega), \quad N_{0} \in L^{\infty}(\Omega) \cap H^{1}(\Omega), \\
K_{0} \in H^{1}(\Omega), \\
H_{0} \geq C_{H}>0, \quad 0<N_{0} \leq \frac{K_{N}}{2}, \quad 0<K_{0} \leq K_{K}
\end{gathered}
$$

has a unique solution $(H, K) \in(X \times X) \cap(Y \times Y)$ and $N \in Z$.

We set

$$
T:=\prod_{i=1}^{6} T_{i}
$$

with $T_{i} \leq 1$ to be defined below.

In order to prove Theorem 2 we construct a sequence

$$
\begin{gathered}
\left(H^{m}, K^{m}\right)_{m \in \mathbb{N}_{0}} \in(X \times X) \cap(Y \times Y), \\
\left(N^{m}\right)_{m \in \mathbb{N}_{0}} \in Z
\end{gathered}
$$

and prove its convergence towards the weak solution of the system.

Let $\left(H^{0}, K^{0}\right) \in(X \times X) \cap(Y \times Y)$ and $N^{0} \in Z$ be the weak solution to the homogeneous system

$$
\begin{gathered}
\frac{\partial H^{0}}{\partial t}-D_{H} \Delta H^{0}+d_{H} H^{0}=0, \\
\frac{\partial N^{0}}{\partial t}+d_{N} H^{0} N^{0}=0, \\
\frac{\partial K^{0}}{\partial t}-\nabla \cdot\left(D_{K}\left(1-\frac{N^{0}}{K_{N}}\right) \nabla K^{0}\right)=0,
\end{gathered}
$$

while $\left(H^{m}, K^{m}\right)_{m \in \mathbb{N}_{0}} \in(X \times X) \cap(Y \times Y)$ and $\left(N^{m}\right)_{m \in \mathbb{N}_{0}} \in Z$ is the weak solution to

$$
\begin{gathered}
\frac{\partial H^{m+1}}{\partial t}-D_{H} \Delta H^{m+1}+d_{H} H^{m+1}=w_{H} K^{m} \\
\frac{\partial N^{m+1}}{\partial t}+d_{N} H^{m+1} N^{m+1}=w_{N} N^{m}\left(1-\frac{N^{m}}{K_{N}}-\theta \frac{K^{m}}{K_{K}}\right) \\
\frac{\partial K^{m+1}}{\partial t}-\nabla \cdot\left(D_{K}\left(1-\frac{N^{m+1}}{K_{N}}\right) \nabla K^{m+1}\right) \\
=w_{K} K^{m}\left(1-\frac{K^{m}}{K_{K}}\right)
\end{gathered}
$$

with the corresponding initial and boundary conditions (2) and (3).

The existence and uniqueness of the functions $\left(H^{m}\right.$, $\left.K^{m}, N^{m}\right)_{m \in \mathbb{N}_{0}}$ in the above sequence are ensured by the following.

Lemma 3 (properties of the iteration sequence). Under assumptions (10) there exists $T>0$ such that

(i) there exists a unique weak solution to the systems (13)(15) and (16)-(18) with conditions (3) and (2), and for every $m \in \mathbb{N}_{0}$ it holds that

$$
\begin{aligned}
& N^{m}, N_{t}^{m} \in L^{\infty}((0, T] \times \Omega), \\
& L^{2}\left(0, T ; H^{2}(\Omega)\right) \cap L^{\infty}\left(0, T ; H^{1}\right. \\
& H_{t}^{m}, K_{t}^{m} \in L^{2}\left(0, T ; L^{2}(\Omega)\right) ;
\end{aligned}
$$$$
H^{m}, K^{m} \in L^{2}\left(0, T ; H^{2}(\Omega)\right) \cap L^{\infty}\left(0, T ; H^{1}(\Omega)\right),
$$ 
(ii) the functions $H^{m}, N^{m}$, and $K^{m}$ are positive for all $m \in$ $\mathbb{N}_{0}$. Moreover, the following inequalities hold:

$$
\begin{aligned}
& H^{m}(t, \mathbf{x}) \geq C_{H} e^{-d_{H} t}, \quad N^{m}(t, \mathbf{x}) \leq \frac{K_{N}}{2}, \\
& K^{m}(t, \mathbf{x}) \leq K_{K} \quad \text { for a.e. } \mathbf{x} \in \Omega, t \in[0, T] ;
\end{aligned}
$$

(iii) the functions $H^{m}, N^{m}$, and $K^{m}$ satisfy for adequate constants $C(\Omega, T)$ and for all $m \in \mathbb{N}_{0}$ the estimates

$$
\begin{gathered}
\left\|H^{m}\right\|_{X}+\left\|H^{m}\right\|_{L^{2}\left(0, T ; H^{2}(\Omega)\right)} \\
\leq C(\Omega, T)\left(\left\|K_{0}\right\|_{H^{1}(\Omega)}+\left\|H_{0}\right\|_{H^{1}(\Omega)}\right), \\
\left\|N^{m}\right\|_{X}^{2} \leq C(\Omega, T)\left\|N_{0}\right\|_{H^{1}(\Omega)}^{2}, \\
\left\|K^{m}\right\|_{X}+\left\|K^{m}\right\|_{L^{2}\left(0, T ; H^{2}(\Omega)\right)} \leq 2 C(\Omega, T)\left\|K_{0}\right\|_{H^{1}(\Omega)} .
\end{gathered}
$$

Remark 4. From (19) it follows that

$$
N^{m} \in L^{\infty}\left(0, T ; L^{2}(\Omega)\right)
$$

for all $m \in \mathbb{N}_{0}$.

Proof of Lemma 3. We perform mathematical induction with respect to $m$.

Induction Start. The proof of the claims in Lemma 3 for $m=0$ is done separately for each of (13)-(15).

(a) With the substitution

$$
\widetilde{H}^{0}(t, \mathbf{x})=H^{0}(t, \mathbf{x}) e^{d_{H} t}
$$

Equation (13) becomes the heat equation

$$
\widetilde{H}_{t}^{0}-D_{H} \Delta \widetilde{H}^{0}=0
$$

thus by the theory of linear parabolic differential equations (see, e.g., [22]) and with the assumption $H_{0} \in H^{1}(\Omega)$ it follows that there exists a unique solution $H^{0}$ of (13) such that

$$
\begin{gathered}
H^{0} \in L^{2}\left(0, T ; H^{2}(\Omega)\right) \cap L^{\infty}\left(0, T ; H^{1}(\Omega)\right), \\
H_{t}^{0} \in L^{2}\left(0, T ; L^{2}(\Omega)\right) .
\end{gathered}
$$

This weak solution also satisfies

$$
\left\|H^{0}\right\|_{X}+\left\|H^{0}\right\|_{L^{2}\left(0, T ; H^{2}(\Omega)\right)} \leq C(\Omega, T)\left\|H_{0}\right\|_{H^{1}(\Omega)} .
$$

Further it is known (see, e.g., [23]) that the solution of (13) can be written explicitly with respect to the initial condition $H_{0}$ and the heat kernel and it is therefore positive.

(b) Equation (14) is linear and has a positive solution:

$$
N^{0}(t, \mathbf{x})=N_{0} e^{-\int_{0}^{t} d_{N} H^{0}(s, \mathbf{x}) d s}>0
$$

which depends on $H^{0}(t, \mathbf{x})$. It follows immediately that

$$
\begin{aligned}
\left\|N^{0}\right\|_{L^{\infty}\left(0, T ; H^{1}(\Omega)\right)}^{2} & =\left\|N_{0} e^{-\int_{0}^{t} d_{N} H^{0} d t}\right\|_{L^{\infty}\left(0, T ; H^{1}(\Omega)\right)}^{2} \\
& \leq\left\|N_{0}\right\|_{H^{1}(\Omega)}^{2},
\end{aligned}
$$

and thus the estimation (23) for $m=0$ is obtained

The corresponding statement (19) for $N^{0}$ is to be justified below.

(c) In order to prove the claims of Lemma 3 for $K^{0}$ we show first that

$$
\begin{aligned}
& N^{0} \in L^{\infty}((0, T] \times \Omega), \\
& N_{t}^{0} \in L^{\infty}((0, T] \times \Omega) .
\end{aligned}
$$

The former follows from

$$
\begin{aligned}
\left\|N^{0}\right\|_{L^{\infty}((0, T] \times \Omega)} \stackrel{(30)}{=}\left\|N_{0} e^{-\int_{0}^{t} d_{N} H^{0} d t}\right\|_{L^{\infty}((0, T] \times \Omega)} \\
\leq\left\|N_{0}\right\|_{L^{\infty}(\Omega)}<\infty .
\end{aligned}
$$

For $t \geq \delta>0$ it is

$$
\begin{aligned}
& \left\|N_{t}^{0}\right\|_{L^{\infty}((0, T] \times \Omega)} \\
& \quad \stackrel{(30)}{=} d_{N}\left\|N_{0} \cdot e^{-\int_{0}^{t} d_{N} H^{0} d t} \cdot H^{0}\right\|_{L^{\infty}((0, T] \times \Omega)}<\infty .
\end{aligned}
$$

For $t \rightarrow 0$ we can consider (27). For its solution it holds (see, e.g., [22]) that

$$
\lim _{(t, \mathbf{x}) \rightarrow\left(0, \mathbf{x}^{0}\right)} \widetilde{H}^{0}(t, \mathbf{x})=H_{0}\left(\mathbf{x}^{0}\right) \quad \text { for every } \mathbf{x}^{0} \in \Omega .
$$

Therefore,

$$
\lim _{(t, \mathbf{x}) \rightarrow\left(0, \mathbf{x}^{0}\right)} H^{0}(t, \mathbf{x})=\lim _{(t, \mathbf{x}) \rightarrow\left(0, \mathbf{x}^{0}\right)} \widetilde{H}^{0}(t, \mathbf{x}) e^{-d_{H} t}=H_{0}\left(\mathbf{x}^{0}\right)
$$

and finally (33) follows, thus also (19) for $m=0$.

The following proof of (20) and (24) upon starting from (15) relies on Theorem 7.1.5 in Evans [22]. However, that result cannot be directly applied to the present case, since the diffusion coefficient $a(t, \mathbf{x})=D_{K}\left(1-\left(N^{0}(t, \mathbf{x}) / K_{N}\right)\right)$ in (15) depends on time.

Let

$$
k_{m}(t):=\sum_{i=1}^{m} d_{m}^{i}(t) w_{i}
$$

with functions $w_{i}=w_{i}(\mathbf{x})$ such that

$$
\begin{gathered}
\left\{w_{i}\right\}_{i=1}^{\infty} \text { is an orthogonal basis of } H^{1}(\Omega), \\
\left\{w_{i}\right\}_{i=1}^{\infty} \text { is an orthonormal basis of } L^{2}(\Omega) .
\end{gathered}
$$

Considering the symmetric bilinear form

$$
A\left[k_{m}, k_{m}\right]:=\int_{\Omega} a(t, \mathbf{x})\left(\nabla k_{m}\right)^{2} d \mathbf{x}
$$


the dependence of the coefficient $a(t, \mathbf{x})$ on $t$ leads in its time derivative

$$
\begin{aligned}
\frac{d}{d t} A\left[k_{m}, k_{m}\right]= & \int_{\Omega} a^{\prime}(t, \mathbf{x})\left(\nabla k_{m}\right)^{2} d \mathbf{x} \\
& +2 \int_{\Omega} a(t, \mathbf{x})\left(\nabla k_{m}\right)^{\prime} \nabla k_{m} d \mathbf{x}
\end{aligned}
$$

to a supplementary summand

$$
\int_{\Omega} a^{\prime}(t, \mathbf{x})\left(\nabla k_{m}\right)^{2} d \mathbf{x}=-\int_{\Omega} \frac{D_{K}}{K_{N}}\left(N^{0}\right)^{\prime}(t, \mathbf{x})\left(\nabla k_{m}\right)^{2} d \mathbf{x},
$$

where for shortness we denoted by ${ }^{\prime}$ the derivative with respect to $t$.

The rest of the proof of Theorem 7.1.5 in [22] can now be adapted to obtain for an arbitrary $\zeta>0$ the estimate

$$
\begin{aligned}
\left\|k_{m}^{\prime}\right\|_{L^{2}(\Omega)}^{2} & +\frac{d}{d t}\left(\frac{1}{2} A\left[k_{m}, k_{m}\right]\right) \\
\leq & \frac{C}{\zeta}\left(\left\|k_{m}\right\|_{H^{1}(\Omega)}^{2}+\|f\|_{L^{2}(\Omega)}^{2}\right) \\
& +2 \zeta\left\|k_{m}^{\prime}\right\|_{L^{2}(\Omega)}^{2}+\frac{1}{2} \int_{\Omega} \frac{D_{K}}{K_{N}}\left(N^{0}\right)^{\prime}\left(\nabla k_{m}\right)^{2} d \mathbf{x} .
\end{aligned}
$$

Now let (recall (33))

$$
M_{N^{0}}:=\frac{D_{K}}{K_{N}}\left\|N_{t}^{0}\right\|_{L^{\infty}((0, T] \times \Omega)} .
$$

Upon integrating with respect to $t$ one can majorize

$$
\begin{aligned}
\int_{0}^{T} \int_{\Omega} \frac{D_{K}}{K_{N}}\left(N^{0}\right)^{\prime}\left(\nabla k_{m}\right)^{2} d \mathbf{x} d t & \leq M_{N^{0}} \int_{0}^{T}\left\|\nabla k_{m}\right\|_{L^{2}(\Omega)}^{2} d t \\
& \leq M_{N^{0}}\left\|k_{m}\right\|_{L^{2}\left(0, T ; H^{1}(\Omega)\right)}^{2} \\
& \leq \gamma(\Omega, T)<\infty,
\end{aligned}
$$

with $\gamma(\Omega, T)$ an adequate constant. The rest of the proof can be done as in Theorem 7.1.5 in [22], upon taking into account (32) and $K_{0} \in H^{1}(\Omega)$ in order to show that there exists a unique weak solution $K^{0}(t, \mathbf{x})$ to $(15)$ such that

$$
\begin{gathered}
K^{0} \in L^{2}\left(0, T ; H^{2}(\Omega)\right) \cap L^{\infty}\left(0, T ; H^{1}(\Omega)\right), \\
K_{t}^{0} \in L^{2}\left(0, T ; L^{2}(\Omega)\right), \\
\left\|K^{0}\right\|_{X}+\left\|K^{0}\right\|_{L^{2}\left(0, T ; H^{2}(\Omega)\right)} \leq C(\Omega, T)\left\|K_{0}\right\|_{H^{1}(\Omega)} .
\end{gathered}
$$

Since

$$
K_{0}^{0}(\mathbf{x})>0
$$

it follows from the weak maximum principle that $K^{0}(t, \mathbf{x})>0$ and thus also the positivity of $K^{0}(t, \mathbf{x})$.

The proof of the inequalities (21) for $m=0$ does not differ from the one for a general $m \in \mathbb{N}$ given below and is therefore omitted here.
With (a)-(c) we proved all statements of Lemma 3 for $m=0$.

Induction Hypothesis. Assume the assertions of the lemma hold for an arbitrary $m \in \mathbb{N}_{0}$.

Inductive Step. The proof for $m+1$ is to be done separately for each of (16)-(18). Since for a corresponding embedding constant $c_{1}:=c_{1}(\Omega, T)$

$$
\begin{aligned}
\int_{0}^{T}\left\|K^{m}\right\|_{L^{2}(\Omega)}^{2} d t \leq c_{1} \int_{0}^{T}\left\|K^{m}\right\|_{H^{1}(\Omega)}^{2} d t \\
\underset{\substack{\leq \\
\text { ind. hyp. }}}{\leq} 4 c_{1} C^{2}(\Omega, T) T\left\|K_{0}\right\|_{H^{1}(\Omega)}^{2}<\infty
\end{aligned}
$$

and thus

$$
K^{m} \in L^{2}\left(0, T ; L^{2}(\Omega)\right),
$$

the existence of a unique weak solution to (16), (2), and (3) follows from the theory of linear parabolic differential equations. The solution $H^{m+1}(t, \mathbf{x})$ satisfies

$$
\begin{gathered}
H^{m+1} \in L^{2}\left(0, T ; H^{2}(\Omega)\right) \cap L^{\infty}\left(0, T ; H^{1}(\Omega)\right), \\
H_{t}^{m+1} \in L^{2}\left(0, T ; L^{2}(\Omega)\right), \\
\left\|H^{m+1}\right\|_{X}+\left\|H^{m+1}\right\|_{L^{2}\left(0, T ; H^{2}(\Omega)\right)} \\
\leq C_{1}(\Omega, T)\left(2 w_{H} C(\Omega, T) \sqrt{c_{1} T}\left\|K_{0}\right\|_{H^{1}(\Omega)}+\left\|H_{0}\right\|_{H^{1}(\Omega)}\right) \\
\leq \mathscr{C}(\Omega, T)\left(\left\|K_{0}\right\|_{H^{1}(\Omega)}+\left\|H_{0}\right\|_{H^{1}(\Omega)}\right),
\end{gathered}
$$

with $\mathscr{C}(\Omega, T)=\max \left\{C_{1}(\Omega, T), C_{1}(\Omega, T) 2 w_{H} C(\Omega, T) \sqrt{c_{1} T}\right.$.

In order to establish the lower bound for $H^{m+1}$ define an auxiliary function $\psi^{m+1}(t, \mathbf{x}):=H^{m+1}(t, \mathbf{x})-C_{H} e^{-d_{H} t}$, for which it holds

$$
\begin{aligned}
& \left\langle\psi_{t}^{m+1}(t), \phi\right\rangle+D_{H} \int_{\Omega} \nabla \psi^{m+1} \nabla \phi d \mathbf{x}+d_{H} \int_{\Omega} \psi^{m+1} \phi d \mathbf{x} \\
& =\left\langle w_{H} K^{m}, \phi\right\rangle .
\end{aligned}
$$

For every nonnegative $\phi \in H^{1}(\Omega)$ the right-hand side is positive. Further, $\psi^{m+1}(0, \mathbf{x}) \geq 0$ by construction; thus it follows with the weak maximum principle that $\psi^{m+1} \geq 0$ a.e. which leads to $H^{m+1}(t, \mathbf{x}) \geq C_{H} e^{-d_{H} t}$.

Now (17) is a linear, inhomogeneous differential equation, with solution

$$
N^{m+1}(t, \mathbf{x})=e^{-\alpha(t, \mathbf{x})}\left(N_{0}(\mathbf{x})+\int_{0}^{t} \beta(s, \mathbf{x}) e^{\alpha(s, \mathbf{x})} d s\right),
$$


where

$$
\begin{gathered}
\alpha(t, \mathbf{x})=\int_{0}^{t} d_{N} H^{m+1}(v, \mathbf{x}) d v \\
\beta(s, \mathbf{x})=w_{N} N^{m}(s, \mathbf{x})\left(1-\frac{N^{m}(s, \mathbf{x})}{K_{N}}-\theta \frac{K^{m}(s, \mathbf{x})}{K_{K}}\right) .
\end{gathered}
$$

In order to prove (19) for $m+1$ we have to show that

$$
\begin{aligned}
& N^{m+1} \in L^{\infty}((0, T] \times \Omega), \\
& N_{t}^{m+1} \in L^{\infty}((0, T] \times \Omega) .
\end{aligned}
$$

Obviously, the first assertion (54) holds, due to the induction hypothesis.

Next, estimate

$$
\begin{aligned}
\left\|N_{t}^{m+1}\right\|_{L^{\infty}((0, T] \times \Omega)} & \\
\leq & w_{N}\left\|N^{m}\right\|_{L^{\infty}((0, T] \times \Omega)}\left\|1-\frac{N^{m}}{K_{N}}-\theta \frac{K^{m}}{K_{K}}\right\|_{L^{\infty}((0, T] \times \Omega)} \\
& +d_{N}\left\|N^{m+1}\right\|_{L^{\infty}((0, T] \times \Omega)}\left\|H^{m+1}\right\|_{L^{\infty}((0, T] \times \Omega)}<\infty,
\end{aligned}
$$

due to (19).

Using again the induction hypothesis, the regularity of the initial data, and the properties of the solutions to the heat equations it follows immediately that $\left\|H^{m+1}\right\|_{L^{\infty}((0, T] \times \Omega)}<\infty$, which leads to

$$
\left\|N_{t}^{m+1}\right\|_{L^{\infty}((0, T] \times \Omega)}<\infty
$$

and thus (55) is proved.

Now we prove the positivity of $N^{m+1}$ and the corresponding inequality in (21). To this aim use the induction hypothesis to observe that

$$
\begin{aligned}
N^{m+1}(t, \mathbf{x}) & \leq \frac{K_{N}}{2} e^{-\alpha(t, \mathbf{x})}+\int_{0}^{t} \beta(s, \mathbf{x}) e^{-(\alpha(t, \mathbf{x})-\alpha(s, \mathbf{x}))} d s \\
& \leq \frac{K_{N}}{2} e^{-\alpha(t, \mathbf{x})}+w_{N} \frac{K_{N}}{2} \int_{0}^{t} e^{-(\alpha(t, \mathbf{x})-\alpha(s, \mathbf{x}))} d s .
\end{aligned}
$$

Next notice that there exists a positive constant $\widetilde{C}_{H}$ such that $H^{m+1}(t, \mathbf{x}) \geq \widetilde{C}_{H}$ for a.e. $\mathbf{x} \in \Omega, t \in[0, T]$. This leads to the estimate

$$
\begin{aligned}
N^{m+1}(t, \mathbf{x}) & \leq \frac{K_{N}}{2} e^{-d_{N} \widetilde{C}_{H} t}+w_{N} \frac{K_{N}}{2} \frac{1}{d_{N} \widetilde{C}_{H}}\left(1-e^{-d_{N} \widetilde{C}_{H} t}\right) \\
& \leq \frac{K_{N}}{2}\left(\left(1-\frac{w_{N}}{d_{N} \widetilde{C}_{H}}\right) e^{-d_{N} \widetilde{C}_{H} t}+\frac{w_{N}}{d_{N} \widetilde{C}_{H}}\right) \leq \frac{K_{N}}{2} .
\end{aligned}
$$

This in turn immediately implies via (52) the positivity of $N^{m+1}$.

In the next step we prove the estimate (23) for $N^{m+1}(t, \mathbf{x})$.
Due to (52) we get

$$
\begin{aligned}
\left\|N^{m+1}(t)\right\|_{H^{1}(\Omega)}^{2} & \left\|e^{-\alpha(t)} N_{0}+e^{-\alpha(t)} \int_{0}^{t} \beta(s) e^{\alpha(s)} d s\right\|_{H^{1}(\Omega)}^{2} \\
\leq & 2\left\|N_{0}\right\|_{H^{1}(\Omega)}^{2} \\
& +2 w_{N}^{2}\left\|\int_{0}^{t} N^{m}(s)\left(1-\frac{N^{m}(s)}{K_{N}}-\theta \frac{K^{m}(s)}{K_{K}}\right) d s\right\|_{H^{1}(\Omega)}^{2} \\
\leq & 2\left\|N_{0}\right\|_{H^{1}(\Omega)}^{2} \\
& +2 w_{N}^{2}\left\|\int_{0}^{t}\left(N^{m}(s)-\frac{\left(N^{m}(s)\right)^{2}}{K_{N}^{2}}\right) d s\right\|_{H^{1}(\Omega)}^{2} \\
\leq & 2\left\|N_{0}\right\|_{H^{1}(\Omega)}^{2} \\
& +4 w_{N}^{2}\left(\left\|\int_{0}^{t} N^{m}(s) d s\right\|_{H^{1}(\Omega)}^{2}\right. \\
& \left.+\frac{1}{K_{N}^{2}}\left\|\int_{0}^{t}\left(N^{m}(s)\right)^{2} d s\right\|_{H^{1}(\Omega)}^{2}\right) \\
\leq & \left\|N_{0}\right\|_{H^{1}(\Omega)}^{2}\left[2+4 w_{N}^{2} C(\Omega, T) T^{2}\right] \\
\leq & \mathscr{C}(\Omega, T)\left\|N_{0}\right\|_{H^{1}(\Omega)}^{2}
\end{aligned}
$$

by (23) and the induction hypothesis.

In order to prove the assertions of Lemma 3 for $K^{m+1}(t, \mathbf{x})$ one can apply Theorem 7.1.5 in [22], with (54), (55), and the same justification as for the induction start at (c).

With an adequate embedding constant $c_{2}:=c_{2}(\Omega, T)$

$$
\begin{gathered}
\int_{0}^{T}\left\|K^{m}\left(1-\frac{K^{m}}{K_{K}}\right)\right\|_{L^{2}(\Omega)}^{2} d t \\
\leq \int_{0}^{T}\left(\left\|K^{m}\right\|_{L^{2}(\Omega)}+\left\|\frac{\left(K^{m}\right)^{2}}{K_{K}}\right\|_{L^{2}(\Omega)}\right)^{2} d t \\
\leq 2 \int_{0}^{T}\left\|K^{m}\right\|_{L^{2}(\Omega)}^{2} d t+2 \int_{0}^{T}\left\|\frac{\left(K^{m}\right)^{2}}{K_{K}}\right\|_{L^{2}(\Omega)}^{2} d t \\
\leq 2 c_{1}^{2} \int_{0}^{T}\left\|K^{m}\right\|_{H^{1}(\Omega)}^{2} d t+2 \frac{c_{2}^{4}}{K_{K}^{2}} \int_{0}^{T}\left\|K^{m}\right\|_{H^{1}(\Omega)}^{4} d t \\
\leq 8 c_{1}^{2} C^{2}(\Omega, T)\left\|K_{0}\right\|_{H^{1}(\Omega)}^{2} T_{1} T_{2} \\
\quad+32 \frac{c_{2}^{4}}{K_{K}^{2}} C^{4}(\Omega, T)\left\|K_{0}\right\|_{H^{1}(\Omega)}^{4} T_{1} T_{2}<\infty,
\end{gathered}
$$

by (24) and the induction hypothesis; therefore $K^{m}(1-$ $\left.\left(K^{m} / K_{K}\right)\right) \in L^{2}\left(0, T ; L^{2}(\Omega)\right)$ and $K_{0}(\mathbf{x}) \in H^{1}(\Omega)$. By applying 
Theorem 7.1.5 in [22] it follows that (18) has a unique weak solution $K^{m+1}(t, \mathbf{x})$ with

$$
\begin{gathered}
K^{m+1} \in L^{2}\left(0, T ; H^{2}(\Omega)\right) \cap L^{\infty}\left(0, T ; H^{1}(\Omega)\right), \\
K_{t}^{m+1} \in L^{2}\left(0, T ; L^{2}(\Omega)\right) .
\end{gathered}
$$

Now choose $T_{1}$ such that $\max \left\{T_{1} C^{2}(\Omega, T), T_{1} C^{4}(\Omega, T)\right\} \leq 1$ and

$$
T_{2}:=\min \left\{\frac{1}{2}, \frac{1}{16 w_{K}^{2} c_{1}^{2}\left\|K_{0}\right\|}, \frac{K_{K}^{2}}{64 w_{K}^{2} c_{2}^{4}\left\|K_{0}\right\|^{3}}\right\} .
$$

Then

$$
\int_{0}^{T}\left\|w_{K} K^{m}\left(1-\frac{K^{m}}{K_{K}}\right)\right\|_{L^{2}(\Omega)}^{2} d t \leq\left\|K_{0}\right\|_{H^{1}(\Omega)}
$$

and thus the estimate

$$
\left\|K^{m+1}\right\|_{X}+\left\|K^{m+1}\right\|_{L^{2}\left(0, T ; H^{2}(\Omega)\right)} \leq 2 C(\Omega, T)\left\|K_{0}\right\|_{H^{1}(\Omega)}
$$

holds.

In order to prove the positivity of $K^{m+1}$ we introduce an auxiliary function

$$
\xi^{m+1}(t, \mathbf{x}):=-A t \exp (1-\eta t)+K_{K}-K^{m+1}(t, \mathbf{x}),
$$

for $A$ positive and large enough and $\eta$ a positive constant to be correspondingly chosen (see below). With the aid of this function we show that for all $m \in \mathbb{N}_{0}$

$$
K^{m+1} \leq K_{K}
$$

on an adequate time interval.

\section{Proof (of the Statement (67))}

Induction Start. The proof of (67) for $m=0$ is identical to the one for $m+1$.

Induction Hypothesis. Assume assertion (67) holds for an arbitrary $m \in \mathbb{N}_{0}$.

Inductive Step. Upon using (66) in (18) we get

$$
\begin{aligned}
\frac{\partial \xi^{m+1}}{\partial t} & -\nabla \cdot\left(D_{K}\left(1-\frac{N^{m+1}}{K_{N}}\right) \nabla \xi^{m+1}\right) \\
& =A(\eta t-1) \exp (1-\eta t)-w_{K} K^{m}\left(1-\frac{K^{m}}{K_{K}}\right) .
\end{aligned}
$$

Since

$$
K^{m}\left(1-\frac{K^{m}}{K_{K}}\right) \leq K_{K}
$$

for the right-hand side of (68) we have that

$$
A(\eta t-1) \exp (1-\eta t)-w_{K} K^{m}\left(1-\frac{K^{m}}{K_{K}}\right) \geq 0
$$

holds for $t<T_{3}$ with correspondingly chosen $T_{3}$ and $\eta$ such that $\eta t>1$.

Since by construction $\xi^{m+1}(0, \mathbf{x}) \geq 0$, we can apply the weak maximum principle for $t \leq T_{3}$ to show that

$$
A(\eta t-1) \exp (1-\eta t)+K_{K}-K^{m+1}(t, \mathbf{x})=\xi^{m+1} \geq 0
$$

from which it also follows that

$$
K^{m+1} \leq K_{K}
$$

This completes the proof of the statement (67).

In virtue of (67), for $T \leq 1 / w_{K}$ the right-hand side in (18) is positive. Since by hypothesis $K_{0}^{m+1}>0$, the weak maximum principle implies the positivity of $K^{m+1}$. This ends the proof of all statements in Lemma 3 for an arbitrary $m \in \mathbb{N}_{0}$ and therefore the proof of the lemma itself.

Now we are able to pass to the following.

\section{Proof (of Theorem 2)}

Existence. In order to prove the existence of a weak solution to (1) and (2) we show that the iterative sequence $\left(N^{m}, K^{m}, H^{m}\right)_{m \in \mathbb{N}_{0}}$ is Cauchy.

Due to the completeness of $H^{1}(\Omega)$ and $L^{2}(\Omega)$, this will imply the convergence of the sequence to some limit functions $N, K$, and $H$, these being solutions to (1) and (2).

Consider an arbitrary $m \in \mathbb{N}_{0}$. Since $H_{0}^{m}, H_{0}^{m+1} \in H^{1}(\Omega)$, and $K^{m}, K^{m+1} \in L^{2}\left(0, T ; L^{2}(\Omega)\right)$, it follows that

$$
\begin{gathered}
H_{0}^{m+1}-H_{0}^{m} \in H^{1}(\Omega), \\
K^{m+1}-K^{m} \in L^{2}\left(0, T ; L^{2}(\Omega)\right) .
\end{gathered}
$$

Next, one can apply Theorem 7.1.5 in [22] to the difference $H^{m+1}-H^{m}$ to deduce the estimate

$$
\left\|H^{m+1}-H^{m}\right\|_{X}^{2} \leq C(\Omega, T) \int_{0}^{T}\left\|w_{H} K^{m}-w_{H} K^{m-1}\right\|_{L^{2}(\Omega)}^{2} d t .
$$

The right-hand side above can be further estimated and with the embedding constant $c_{3}:=c_{3}(\Omega, T)$ it follows that

$$
\begin{aligned}
\left\|H^{m+1}-H^{m}\right\|_{X}^{2} & \leq C(\Omega, T) w_{H}^{2} c_{3}^{2} \int_{0}^{T}\left\|K^{m}-K^{m-1}\right\|_{H^{1}(\Omega)}^{2} d t \\
& \leq C(\Omega, T) w_{H}^{2} c_{3}^{2} T_{4}\left\|K^{m}-K^{m-1}\right\|_{X}^{2} \\
& \leq \frac{1}{2}\left\|K^{m}-K^{m-1}\right\|_{X^{\prime}}^{2}
\end{aligned}
$$

where

$$
T_{4}=\min \left\{\frac{1}{4}, \frac{1}{4 C(\Omega, T) w_{H}^{2} c_{3}^{2}}\right\} .
$$


In order to obtain a corresponding estimate for the sequence $\left(N^{m}\right)_{m \in \mathbb{N}}$, consider two consecutive terms in (17) written for $N^{m}$ and $N^{m+1}$ and substract. This leads to

$$
\begin{array}{r}
\frac{\partial}{\partial t}\left(N^{m+1}-N^{m}\right)+d_{N}\left(H^{m+1} N^{m+1}-H^{m} N^{m}\right) \\
=w_{N}\left(N^{m}\left(1-\frac{N^{m}}{K_{N}}-\frac{K^{m}}{K_{K}}\right)\right. \\
\left.-N^{m-1}\left(1-\frac{N^{m-1}}{K_{N}}-\frac{K^{m-1}}{K_{K}}\right)\right) .
\end{array}
$$

Denote $h\left(N^{m}, N^{m-1}\right):=w_{N}\left(N^{m}\left(1-\left(N^{m} / K_{N}\right)-\left(K^{m} / K_{K}\right)\right)-\right.$ $\left.N^{m-1}\left(1-\left(N^{m-1} / K_{N}\right)-\left(K^{m-1} / K_{K}\right)\right)\right)$.

Now multiply with $\left(N^{m+1}-N^{m}\right)$ and integrate with respect to $\mathbf{x}$ to infer

$$
\begin{aligned}
\frac{1}{2} \int_{\Omega} \frac{\partial}{\partial t}( & \left.N^{m+1}-N^{m}\right)^{2} d \mathbf{x} \\
& +d_{N} \int_{\Omega}\left(N^{m+1}-N^{m}\right)^{2} H^{m+1} d \mathbf{x} \\
= & \int_{\Omega}\left(h\left(N^{m}, N^{m-1}\right)-d_{N} N^{m}\left(H^{m+1}-H^{m}\right)\right) \\
& \times\left(N^{m+1}-N^{m}\right) d \mathbf{x} .
\end{aligned}
$$

Thus

$$
\begin{gathered}
\frac{d}{d t}\left\|N^{m+1}-N^{m}\right\|_{L^{2}(\Omega)}^{2} \\
\leq 2 w_{N} \int_{\Omega} \mid\left(N^{m}\left(1-\frac{N^{m}}{K_{N}}-\frac{K^{m}}{K_{K}}\right)\right. \\
\left.-N^{m-1}\left(1-\frac{N^{m-1}}{K_{N}}-\frac{K^{m-1}}{K_{K}}\right)\right) \\
\times\left(N^{m+1}-N^{m}\right) \mid d \mathbf{x} \\
+2 d_{N} \int_{\Omega}\left|N^{m}\left(H^{m+1}-H^{m}\right)\left(N^{m+1}-N^{m}\right)\right| d \mathbf{x} \\
\quad\left[2 w_{N} \|^{m}\left(1-\frac{N^{m}}{K_{N}}-\frac{K^{m}}{K_{K}}\right)\right. \\
-N^{m-1}\left(1-\frac{N^{m-1}}{K_{N}}-\frac{K^{m-1}}{K_{K}}\right) \|_{L^{2}(\Omega)} \\
\left.+2 d_{N}\left\|N^{m}\left(H^{m+1}-H^{m}\right)\right\|_{L^{2}(\Omega)}\right] \\
\times N^{m+1}-N^{m} \|_{L^{2}(\Omega)}
\end{gathered}
$$

Next we estimate the above terms.
TABLE 1: Parameter values used in the model.

\begin{tabular}{lc}
\hline Parameters & Range \\
\hline$K_{N}$ & $5 \times 10^{7} / \mathrm{cm}^{3}$ \\
$K_{K}$ & $5 \times 10^{7} / \mathrm{cm}^{3}$ \\
$w_{N}$ & $1 \times 10^{-6} / \mathrm{s}$ \\
$w_{K}$ & $1 \times 10^{-6} / \mathrm{s}$ \\
$D_{K}$ & $2 \times 10^{-10} / \mathrm{cm}^{2} / \mathrm{s}$ \\
$D_{H}$ & $5 \times 10^{-6} / \mathrm{cm}^{2} / \mathrm{s}$ \\
$w_{H}$ & $2.2 \times 10^{-17} \mathrm{M} \cdot \mathrm{cm}^{3} / \mathrm{s}$ \\
$d_{H}$ & $1.1 \times 10^{-4} / \mathrm{s}$ \\
$d_{N}$ & $0 \rightarrow 10 / \mathrm{M} \cdot \mathrm{s}$ \\
\hline
\end{tabular}

Let (recall (19))

$$
\begin{aligned}
M_{\max }:=\max \left\{M_{N^{m}}:=\left\|N^{m}\right\|_{L^{\infty}((0, T] \times \Omega)},\right. \\
\left.N_{N^{m-1}}:=\left\|N^{m-1}\right\|_{L^{\infty}((0, T] \times \Omega)}\right\} .
\end{aligned}
$$

With the embedding constant $c_{4}:=c_{4}(\Omega, T)$ we obtain for the first term on the right-hand side of (79)

$$
2 w_{N} \| N^{m}-N^{m-1}-\frac{\left(N^{m}\right)^{2}}{K_{N}}+\frac{\left(N^{m-1}\right)^{2}}{K_{N}}
$$

$$
\begin{aligned}
& -\frac{N^{m} K^{m}}{K_{K}}+\frac{N^{m-1} K^{m-1}}{K_{K}} \|_{L^{2}(\Omega)} \\
\leq & 2 w_{N}\left\|N^{m}-N^{m-1}\right\|_{L^{2}(\Omega)} \\
& +\frac{4 w_{N} M_{\max }}{K_{N}}\left\|N^{m}-N^{m-1}\right\|_{L^{2}(\Omega)} \\
& +2 \frac{w_{N} K_{N}}{K_{K}}\left\|K^{m}-K^{m-1}\right\|_{L^{2}(\Omega)} \\
& +2 w_{N}\left\|N^{m}-N^{m-1}\right\|_{L^{2}(\Omega)} \\
\leq & C_{\widetilde{N}}\left\|N^{m}-N^{m-1}\right\|_{L^{2}(\Omega)} \\
& +C_{\widetilde{K}}\left\|K^{m}-K^{m-1}\right\|_{L^{2}(\Omega)}
\end{aligned}
$$

with $C_{\widetilde{N}}:=4 w_{N}\left(1+\left(M_{\max } / K_{N}\right)\right)$ and $C_{\widetilde{K}}:=2 w_{N} K_{N} / K_{K}$.

Now for the second term on the right-hand side of (79)

$$
\begin{aligned}
2 d_{N} \| & N^{m}\left(H^{m+1}-H^{m}\right) \|_{L^{2}(\Omega)} \\
& \leq d_{N} c_{4} \frac{K_{N}}{2}\left\|H^{m+1}-H^{m}\right\|_{H^{1}(\Omega)} \\
& =C_{\widetilde{H}}\left\|H^{m+1}-H^{m}\right\|_{H^{1}(\Omega)},
\end{aligned}
$$




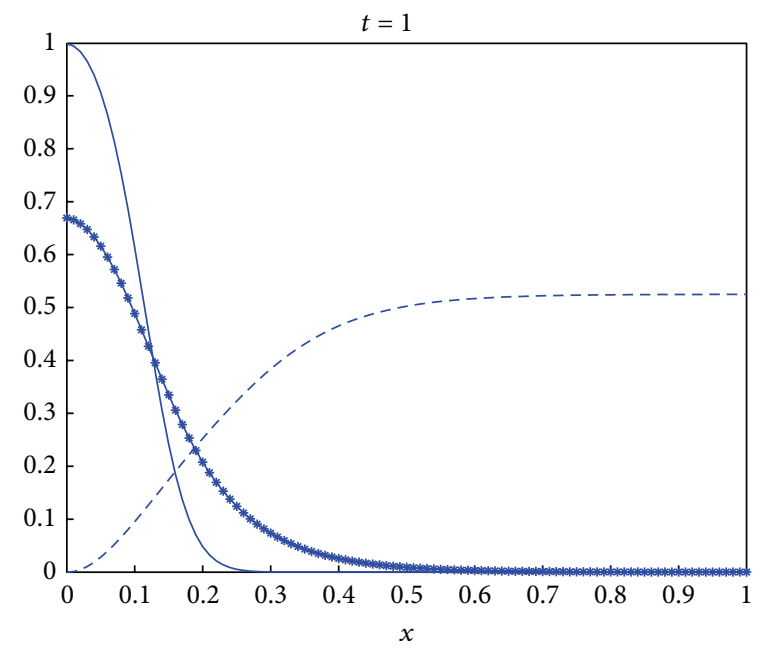

(a)

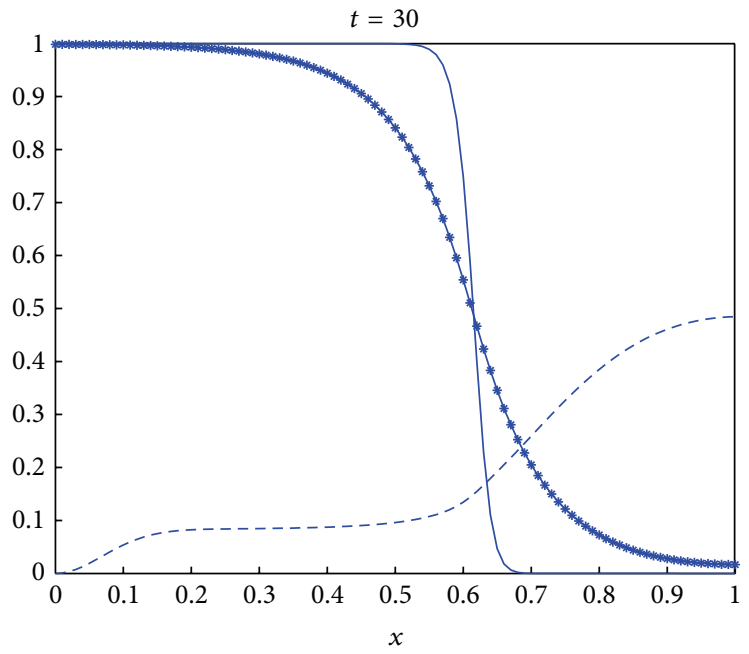

(c)

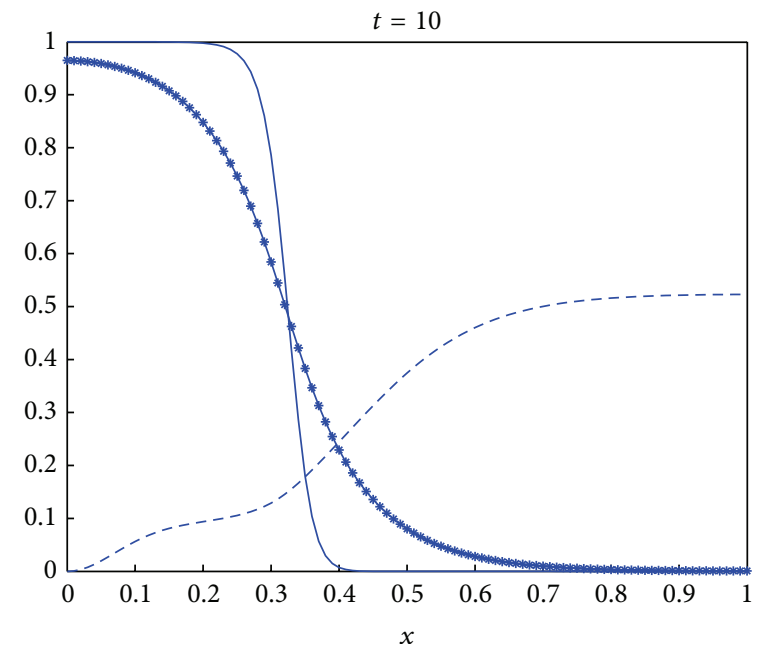

(b)

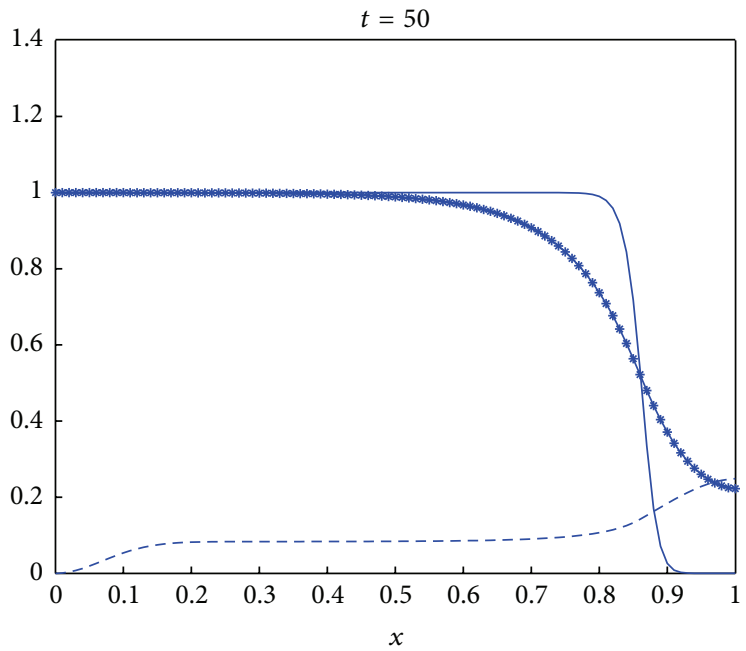

(d)

FIgURE 1: Variations of $\mathrm{H}^{+}$protons (starred), normal tissue (dashed), and neoplastic tissue (solid) for an aggressive tumor.

with $C_{\widetilde{H}}:=d_{N} K_{N} c_{4}$. The two estimates above thus lead to

$$
\begin{gathered}
\frac{d}{d t}\left\|N^{m+1}-N^{m}\right\|_{L^{2}(\Omega)}^{2} \\
\leq \frac{1}{2}\left(C_{\widetilde{N}}\left\|N^{m}-N^{m-1}\right\|_{L^{2}(\Omega)}\right. \\
+C_{\widetilde{K}}\left\|K^{m}-K^{m-1}\right\|_{L^{2}(\Omega)} \\
\left.+C_{\widetilde{H}}\left\|H^{m+1}-H^{m}\right\|_{H^{1}(\Omega)}\right)^{2} \\
+\frac{1}{2}\left\|N^{m+1}-N^{m}\right\|_{L^{2}(\Omega)}^{2} \\
\leq C_{\widetilde{N}}^{2}\left\|N^{m}-N^{m-1}\right\|_{L^{2}(\Omega)}^{2} \\
+C_{\widetilde{K}}^{2}\left\|K^{m}-K^{m-1}\right\|_{L^{2}(\Omega)}^{2}
\end{gathered}
$$

$$
\begin{aligned}
& +C_{\widetilde{H}}^{2}\left\|H^{m+1}-H^{m}\right\|_{H^{1}(\Omega)}^{2} \\
& +\frac{1}{2}\left\|N^{m+1}-N^{m}\right\|_{L^{2}(\Omega)}^{2} .
\end{aligned}
$$

Applying Gronwall's inequality we deduce

$$
\begin{aligned}
&\left\|N^{m+1}-N^{m}\right\|_{L^{2}(\Omega)}^{2} \\
& \leq e^{t / 2} \int_{0}^{t}\left(C_{\widetilde{N}}^{2}\left\|N^{m}-N^{m-1}\right\|_{L^{2}(\Omega)}^{2}\right. \\
&+C_{\widetilde{K}}^{2}\left\|K^{m}-K^{m-1}\right\|_{L^{2}(\Omega)}^{2} \\
&\left.+C_{\widetilde{H}}^{2}\left\|H^{m+1}-H^{m}\right\|_{H^{1}(\Omega)}^{2}\right) d s,
\end{aligned}
$$




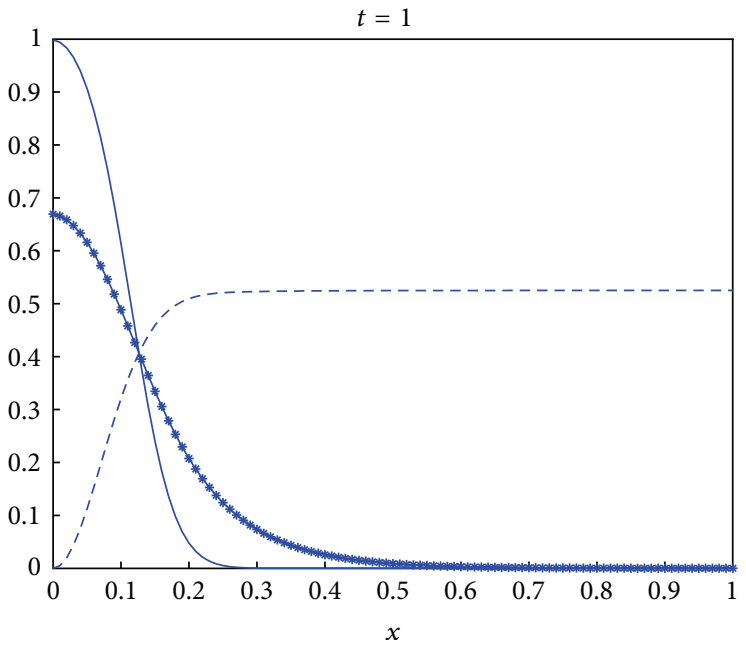

(a)

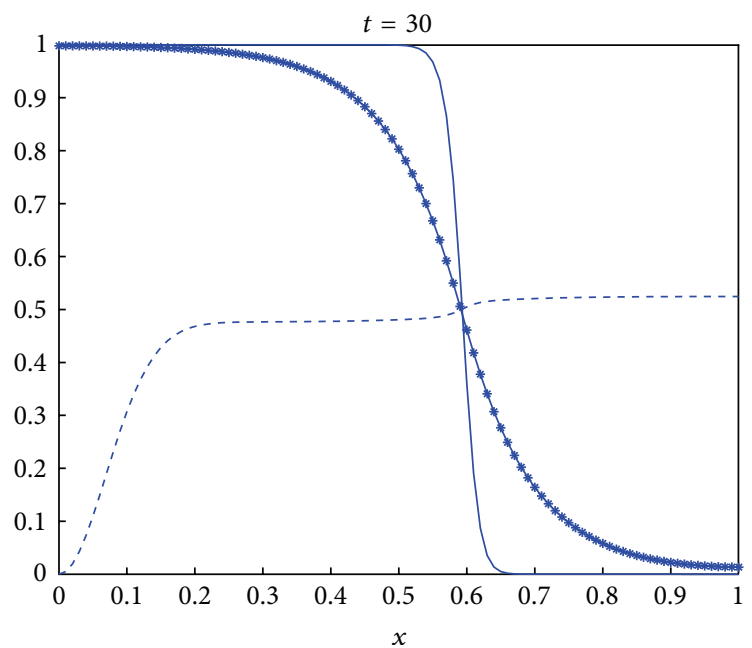

(c)

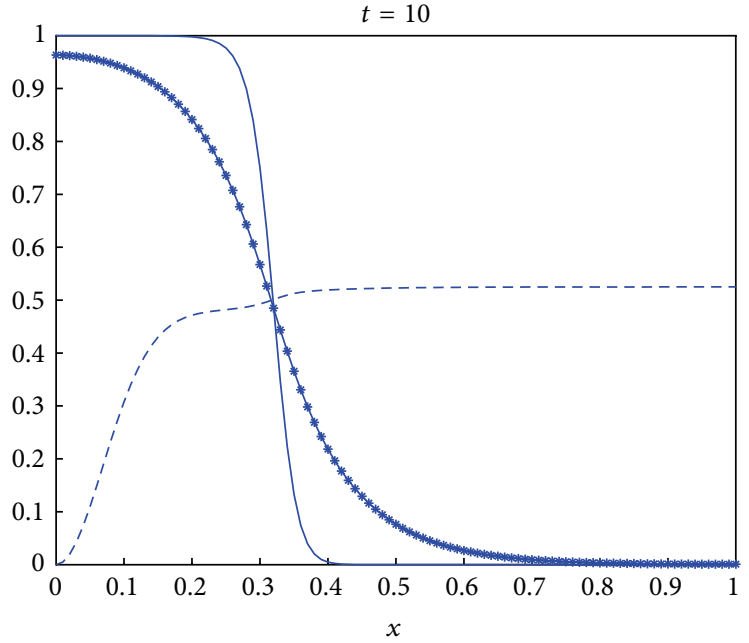

(b)

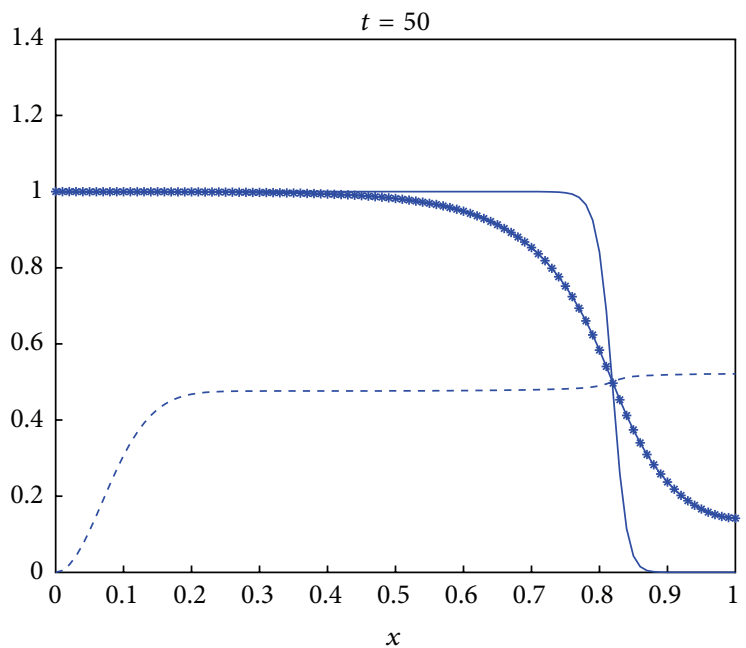

(d)

FIGURE 2: Variations of $\mathrm{H}^{+}$protons (starred), normal tissue (dashed), and neoplastic tissue (solid) for a less-aggressive tumor comparing to the one in Figure 1.

and finally with $D(\Omega, T)=e^{T / 2} \max \left\{C_{\widetilde{N}}^{2}, C_{\widetilde{K}}^{2}, C_{\widetilde{H}}^{2}\right\}$ we get

$$
\begin{gathered}
\left\|N^{m+1}-N^{m}\right\|_{L^{\infty}\left(0, T ; L^{2}(\Omega)\right)}^{2} \\
\leq D(\Omega, T)\left(\left\|N^{m}-N^{m-1}\right\|_{L^{\infty}\left(0, T ; L^{2}(\Omega)\right)}^{2}\right. \\
+\left\|K^{m}-K^{m-1}\right\|_{L^{\infty}\left(0, T ; L^{2}(\Omega)\right)}^{2} \\
\left.+\left\|H^{m+1}-H^{m}\right\|_{X}^{2}\right) T_{5} \\
\leq \frac{1}{4}\left(\left\|N^{m}-N^{m-1}\right\|_{L^{\infty}\left(0, T ; L^{2}(\Omega)\right)}^{2}\right. \\
+\left\|H^{m+1}-H^{m}\right\|_{X}^{2} \\
\left.+\left\|K^{m}-K^{m-1}\right\|_{L^{\infty}\left(0, T ; L^{2}(\Omega)\right)}^{2}\right)
\end{gathered}
$$

$T_{5}$ is chosen such that

$$
D(\Omega, T) T_{5} \leq \frac{1}{4}
$$

Now since $K_{0}^{m}, K_{0}^{m+1} \in H^{1}(\Omega)$ and $K^{m}\left(1-\left(K^{m} / K_{K}\right)\right)$, $K^{m+1}\left(1-\left(K^{m+1} / K_{K}\right)\right) \in L^{2}\left(0, T ; L^{2}(\Omega)\right)$, we get

$$
\begin{gathered}
K_{0}^{m+1}-K_{0}^{m} \in H^{1}(\Omega), \\
{\left[w_{K} K^{m+1}\left(1-\frac{K^{m+1}}{K_{K}}\right)-w_{K} K^{m}\left(1-\frac{K^{m}}{K_{K}}\right)\right]} \\
\in L^{2}\left(0, T ; L^{2}(\Omega)\right) .
\end{gathered}
$$




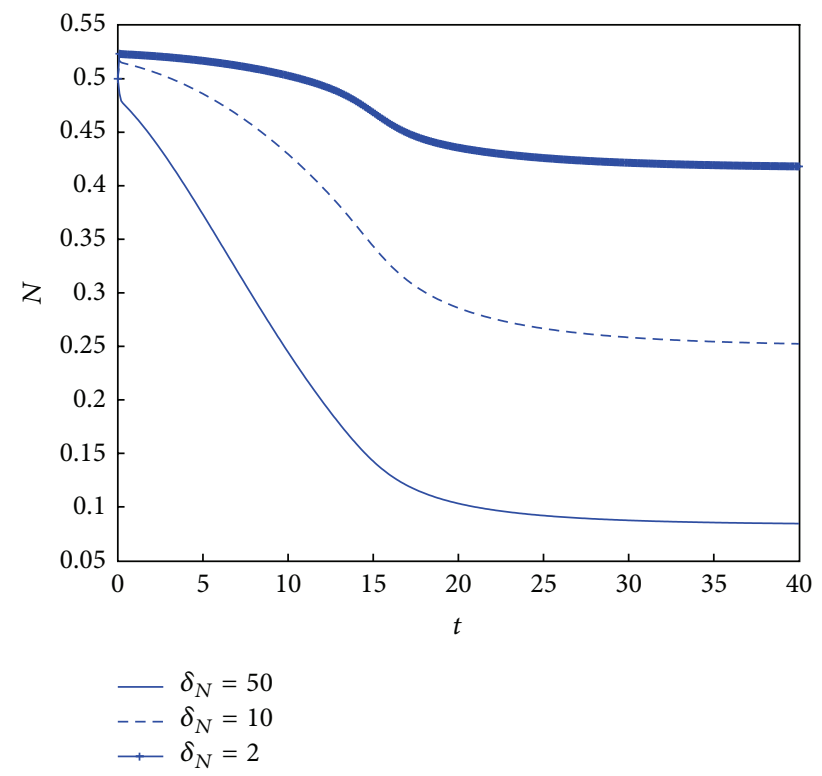

(a)

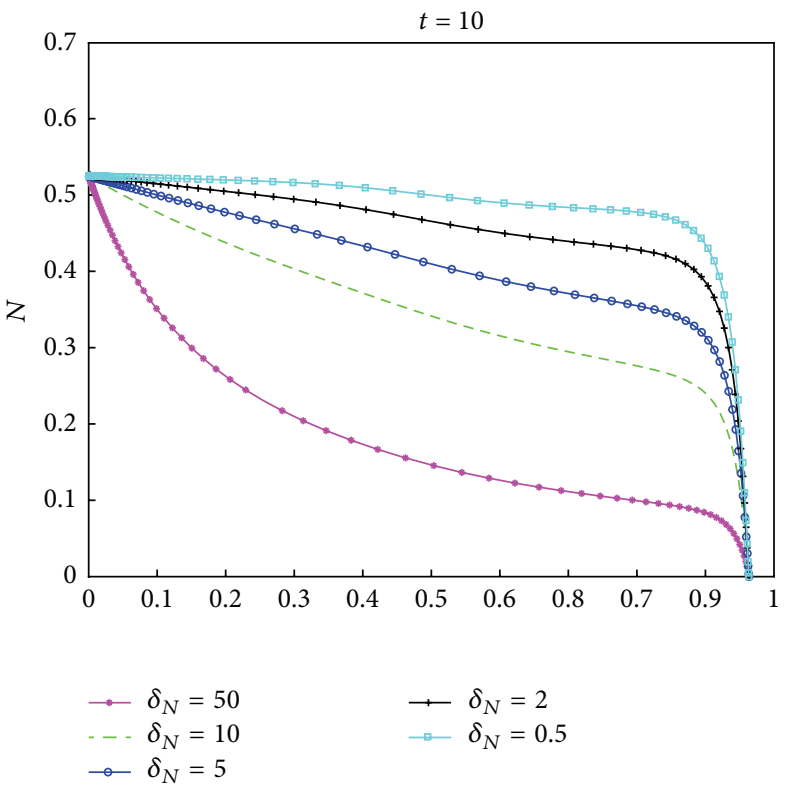

(b)

Figure 3: (a) Evolution of the normal cell density for several different values of $\delta_{N}$. (b) Normal cell density with respect to the $\mathrm{H}^{+}$proton concentration for several different values of $\delta_{N}$.

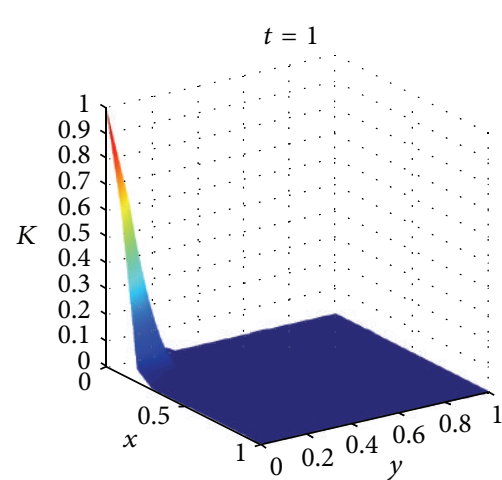

(a)

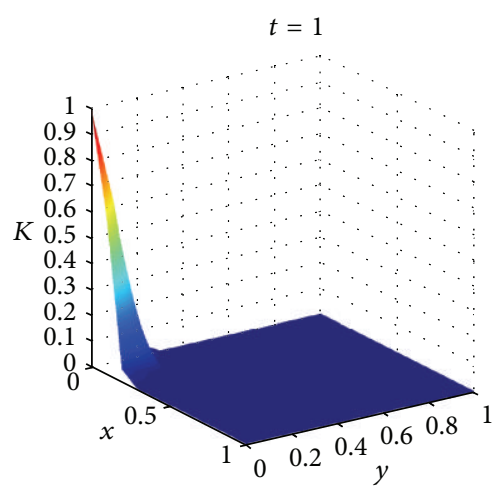

(d)

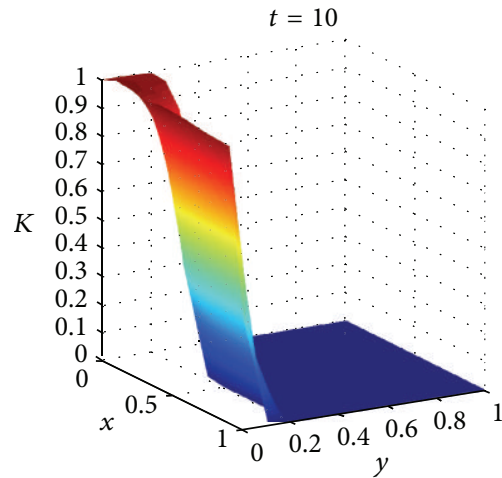

(b)

$t=10$

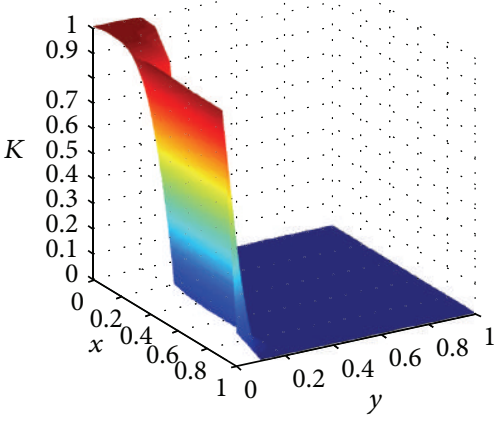

(e)

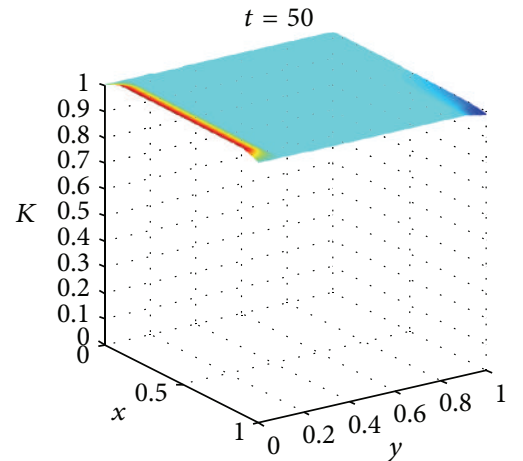

(c)

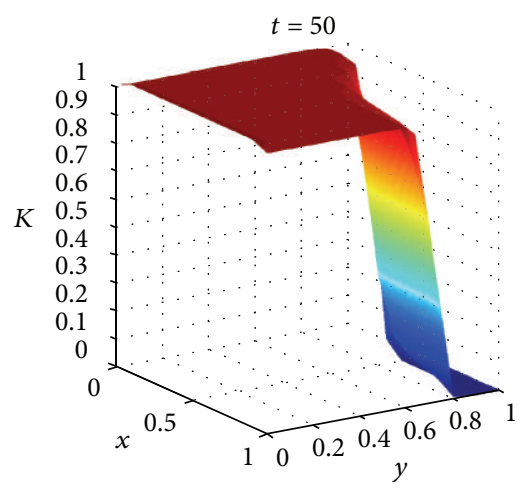

(f)

FIGURE 4: Variations of cancer cells for the cases of an aggressive ((a)-(c)) and a less-aggressive ((d)-(f)) tumor. 


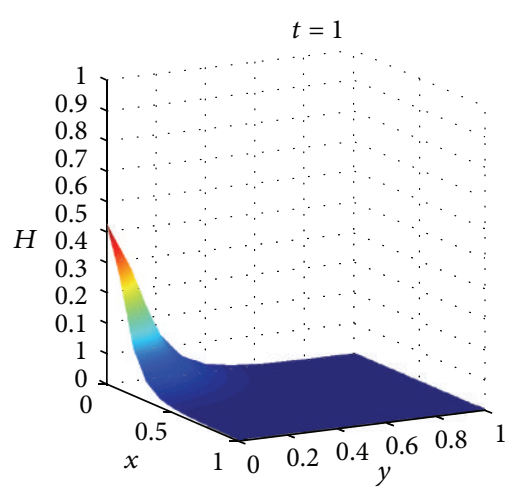

(a)

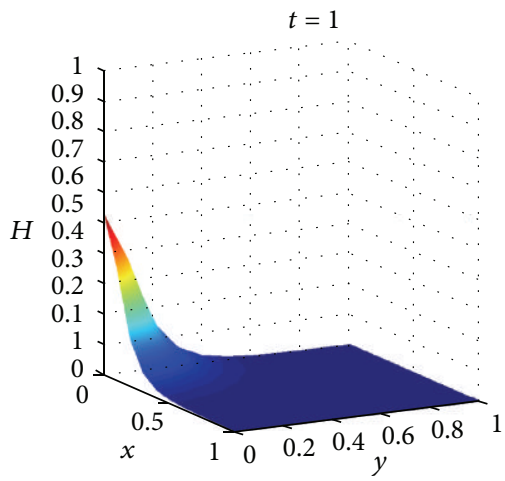

(d)

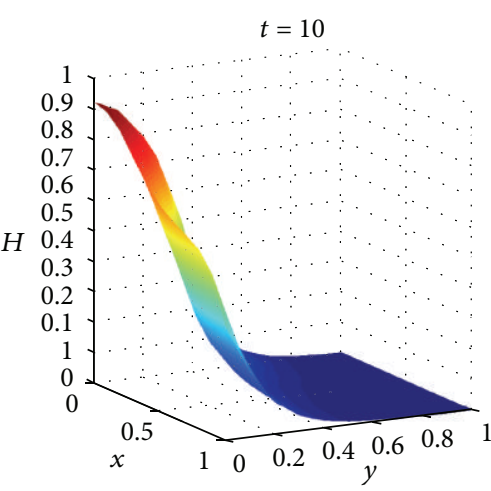

(b)

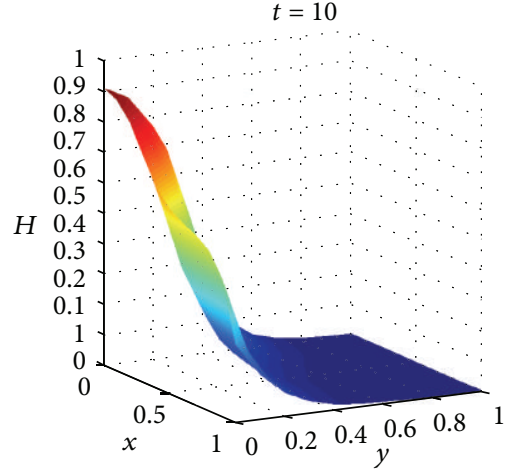

(e)

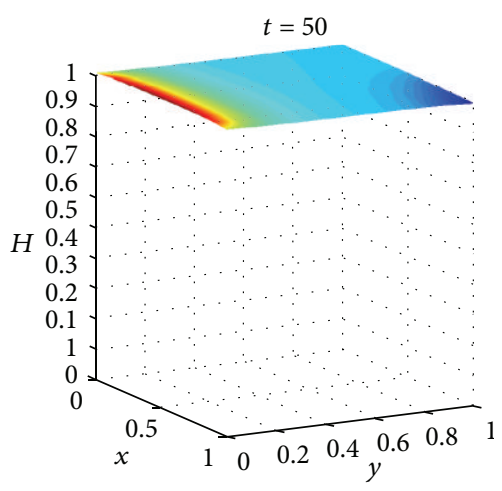

(c)

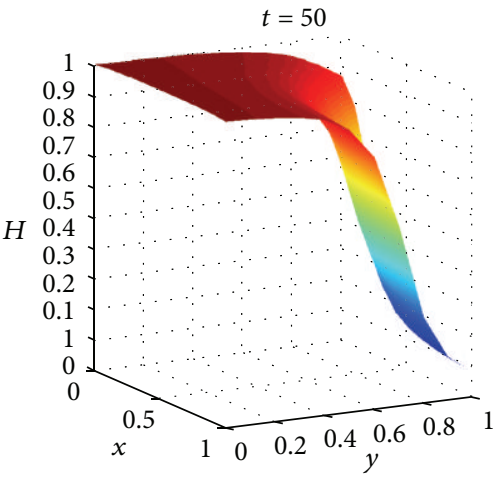

(f)

FIGURE 5: Variations of proton concentration for the cases of an aggressive ((a)-(c)) and a less-aggressive ((d)-(f)) tumor.

Theorem 7.1.5 in [22] can be applied to the difference $K^{m+1}-$ $K^{m}$, leading to

$$
\begin{aligned}
\left\|K^{m+1}-K^{m}\right\|_{X}^{2} & \\
\leq C(\Omega, T) \int_{0}^{T} \| & w_{K} K^{m}\left(1-\frac{K^{m}}{K_{K}}\right) \\
& -w_{K} K^{m-1}\left(1-\frac{K^{m-1}}{K_{K}}\right) \|_{L^{2}(\Omega)}^{2} d t .
\end{aligned}
$$

The right hand side of this inequality can further be majorized and with the embedding constants $c_{5}:=\mathcal{c}_{5}(\Omega, T)$ and $c_{6}:=$ $c_{6}(\Omega, T)$ it follows that

$$
\begin{gathered}
\int_{0}^{T}\left\|w_{K} K^{m}\left(1-\frac{K^{m}}{K_{K}}\right)-w_{K} K^{m-1}\left(1-\frac{K^{m-1}}{K_{K}}\right)\right\|_{L^{2}(\Omega)}^{2} d t \\
\leq 2 \int_{0}^{T} \frac{w_{K}^{2}}{K_{K}^{2}}\left\|\left(K^{m}\right)^{2}-\left(K^{m-1}\right)^{2}\right\|_{L^{2}(\Omega)}^{2} \\
+w_{K}^{2}\left\|K^{m}-K^{m-1}\right\|_{L^{2}(\Omega)}^{2} d t
\end{gathered}
$$

$$
\begin{aligned}
& \leq 2 \int_{0}^{T} c_{5}^{4} \frac{w_{K}^{2}}{K_{K}^{2}}\left\|K^{m}-K^{m-1}\right\|_{H^{1}(\Omega)}^{2}\left\|K^{m}+K^{m-1}\right\|_{H^{1}(\Omega)}^{2} \\
& \quad+w_{K}^{2} c_{6}^{2}\left\|K^{m}-K^{m-1}\right\|_{H^{1}(\Omega)}^{2} d t \\
& \leq\left(\int _ { 0 } ^ { T } \left(4 c_{5}^{4} \frac{w_{K}^{2}}{K_{K}^{2}}\left[\left\|K^{m}\right\|_{H^{1}(\Omega)}^{2}+\left\|K^{m-1}\right\|_{H^{1}(\Omega)}^{2}\right]\right.\right. \\
& \left.\left.\quad+2 w_{K}^{2} c_{6}^{2}\right) d t\right)\left\|K^{m}-K^{m-1}\right\|_{X}^{2} \\
& \leq\left(32 C^{2}(\Omega, T) c_{5}^{4} \frac{w_{K}^{2}}{K_{K}^{2}} T_{6}\left\|K_{0}\right\|_{H^{1}(\Omega)}^{2}+2 w_{K}^{2} c_{6}^{2} T_{6}\right) \\
& \quad \times\left\|K^{m}-K^{m-1}\right\|_{X}^{2} \\
& \leq \frac{1}{4}\left\|K^{m}-K^{m-1}\right\|_{X}^{2},
\end{aligned}
$$

where

$$
\begin{gathered}
T_{6}:=\min \left\{\frac{1}{8}, \frac{1}{8 \kappa}, \frac{1}{8 \lambda}\right\}, \\
k:=32 C^{2}(\Omega, T) c_{5}^{4} \frac{w_{K}^{2}}{K_{K}^{2}}\left\|K_{0}\right\|_{H^{1}(\Omega)}^{2}, \quad \lambda:=2 w_{K}^{2} c_{6}^{2} .
\end{gathered}
$$




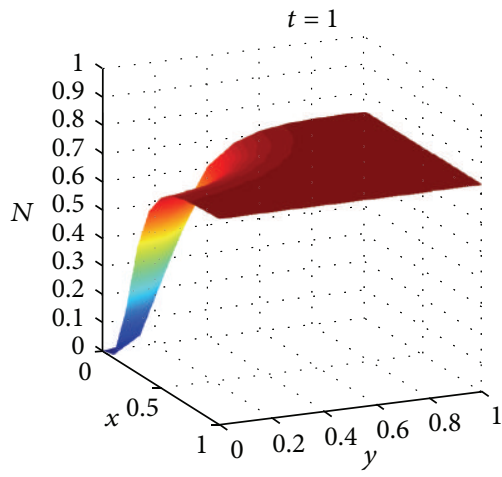

(a)

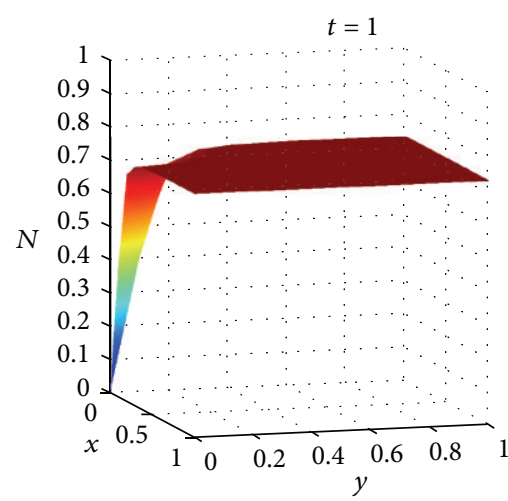

(d)

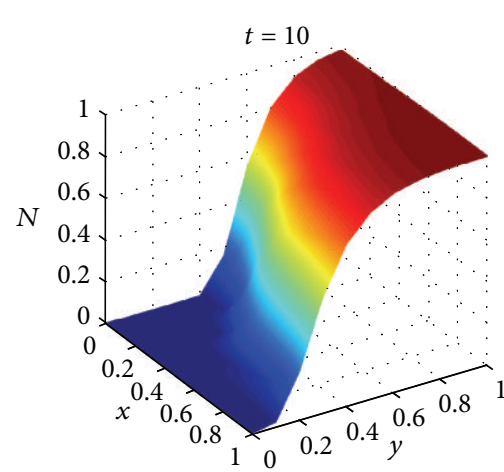

(b)

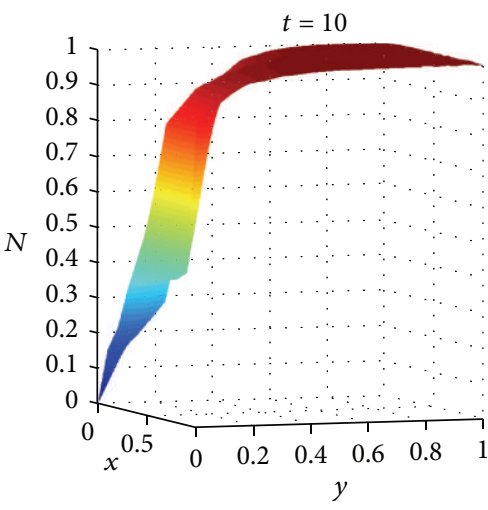

(e)

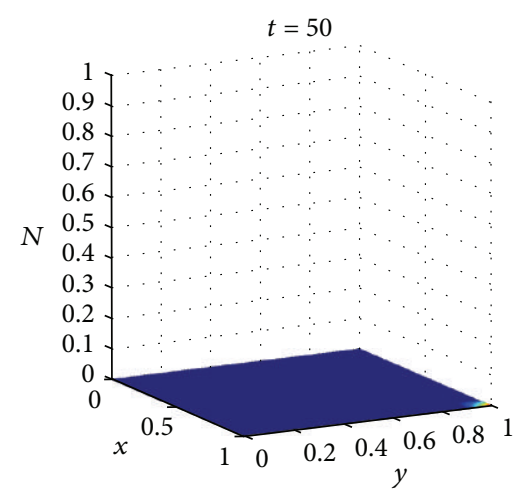

(c)

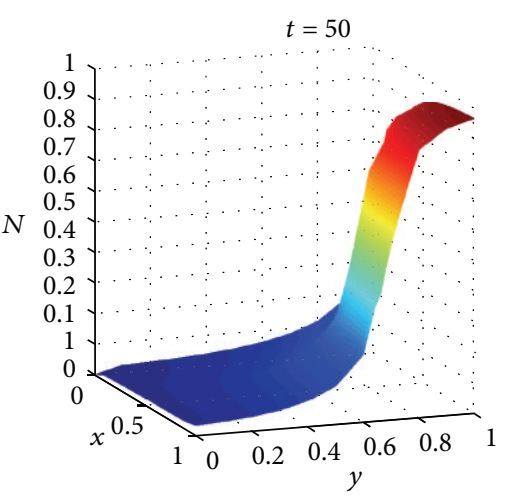

(f)

FIGURE 6: Variations of healthy tissue for the cases of an aggressive ((a)-(c)) and a less-aggressive ((d)-(f)) tumor.

Thus, putting all together,

$$
\begin{gathered}
\left\|K^{m+1}-K^{m}\right\|_{X}^{2}+\left\|N^{m+1}-N^{m}\right\|_{L^{\infty}\left(0, T ; L^{2}(\Omega)\right)}^{2}+\left\|H^{m+1}-H^{m}\right\|_{X}^{2} \\
\leq \frac{1}{4}\left(3\left\|K^{m}-K^{m-1}\right\|_{X}^{2}+\left\|N^{m}-N^{m-1}\right\|_{L^{\infty}\left(0, T ; L^{2}(\Omega)\right)}^{2}\right) .
\end{gathered}
$$

Therefore, $\left(H^{m}, N^{m}, K^{m}\right)$ is a Cauchy sequence in $X \times$ $L^{\infty}\left(0, T ; L^{2}(\Omega)\right) \times X$, from which the existence of a weak solution follows.

Uniqueness. Let $\left(K_{1}, N_{1}, H_{1}\right)$ and $\left(K_{2}, N_{2}, H_{2}\right)$ be two solutions to (1)-(3). Due to the previous estimates

$$
\begin{gathered}
\left\|K_{1}-K_{2}\right\|_{X}^{2} \leq \frac{1}{4}\left\|K_{1}-K_{2}\right\|_{X}^{2}, \\
\left\|H_{1}-H_{2}\right\|_{X}^{2} \leq \frac{1}{4}\left\|K_{1}-K_{2}\right\|_{X}^{2} \\
\left\|N_{1}-N_{2}\right\|_{L^{\infty}\left(0, T ; L^{2}(\Omega)\right)}^{2} \\
\leq \frac{1}{4}\left\|N_{1}-N_{2}\right\|_{L^{\infty}\left(0, T ; L^{2}(\Omega)\right)}^{2}+\frac{1}{4}\left\|H_{1}-H_{2}\right\|_{X}^{2} \\
+\frac{1}{4}\left\|K_{1}-K_{2}\right\|_{X}^{2} ;
\end{gathered}
$$
initially the normal cells are at half of their carrying capacity, while the tumor cells can be close to theirs and thus prone to invade the surrounding tissue. Since the $\mathrm{pH}$ level is lowered

thus $K_{1}=K_{2}$ (92) and with (93) it follows that $H_{1}=H_{2}$. Finally, (94) implies that $N_{1}=N_{2}$. This completes the proof of the uniqueness.

Regularity of the Solution. From (20) it follows that $\left(K^{m}, H^{m}\right)$ is uniformly bounded with respect to $m$ in $Y \times Y$; therefore $Y$ is compactly embedded in $L^{2}\left(0, T ; H^{1}(\Omega)\right)$. This implies that for $m \rightarrow \infty$ we have $(K, H) \in Y \times Y$.

Theorem 5. (The local solution from Theorem 2 exists globally).

The proof follows upon sequentially extending the time interval on which the solution exists: the previously deduced estimates allow for a bootstrap of the local existence proof in a subsequent step on the time interval $[T, 2 T]$, then on $[2 T, T]$, and so forth. Eventually the existence of a unique solution in shown on $[0, \mathbb{T}]$ for any bounded $\mathbb{T}$.

\section{Numerical Simulations}

In this section we perform the numerical simulation of the system (7). The boundary conditions for $K$ and $H$ are the no-flux boundary conditions given by (2). We assume that$$
\text { invade the surrounding tissue. Since the pH level is lowered }
$$ 
by the cancer cells the concentration of protons is taken proportional to the density of the latter. Thus using these assumptions we choose for the initial conditions of the system (7)

$$
\begin{gathered}
K(0, x)=\exp \left(-\frac{|x|^{2}}{\epsilon}\right), \quad \epsilon>0, \\
N(0, x)=0.5 *\left(1-\exp \left(-\frac{|x|^{2}}{\epsilon}\right)\right), \quad \epsilon>0, \\
H(0, x)=\zeta K(0, x), \quad \zeta \in[0,1),
\end{gathered}
$$

where $x \in[0,1]$ and $x \in[0,1] \times[0,1]$ in one- and twodimensional cases, respectively. In our computations we have taken both $\zeta$ and the strength parameter $\theta$ to be 0.5 .

For the discretization of the model the finite difference method is employed. Thereby in the one-dimensional case the interval $[0,1]$ is divided into $m$ parts with $m+1$ nodes, whereas in two dimensions each of the axes is divided into $m$ parts, thus obtaining a $(m+1) \times(m+1)$ mesh in two dimensions. Moreover the nodes are reordered in a row-wise manner, leading to a total of $(m+1)^{2}$ nodes. Thereby the subindex $i$ in the discretized equations denotes the spatial node $x_{i}$, where $i=1,2, \ldots, m+1$ and $i=1,2, \ldots,(m+1)^{2}$ in one and two dimensions, respectively. We use forward differences for the time derivatives in the system. The central difference is used for the diffusion term in the equation characterizing the ion concentration:

$$
\frac{H_{i}^{n+1}-H_{i}^{n}}{\Delta t}=\delta_{H} K_{i}^{n}-\delta_{H} H_{i}^{n+1}+\left(\frac{H_{i-1}^{n+1}-2 H_{i}^{n+1}+H_{i+1}^{n+1}}{\Delta x^{2}}\right)
$$

where $n$ denotes the time level and $\Delta t$ and $\Delta x$ are the time and space increments, respectively. The discretized equations (96) for each $i$ leads to the following system of equations of the form

$$
\mathbf{A}_{\mathbf{H}} \mathbf{H}^{n+1}=\mathbf{H}^{n}+\vartheta_{\mathbf{H}}^{n}
$$

with $\mathbf{H}^{n+1}$ and $\vartheta_{\mathbf{H}}^{n}$ standing for the vectors containing the values of $H$ and $\Delta t \delta_{H} K$ at the $(n+1)$ st and $n$th time levels at the discretized space points, respectively; $\mathbf{A}_{\mathbf{H}}$ being the tridiagonal and block tridiagonal matrix for the one- and twodimensional cases, respectively. The updated values $\mathbf{H}^{n+1}$ are used to find the values of the normal cell density at the time level $n+1$ by solving

$$
N_{i}^{n+1}=\frac{1}{1+\Delta t \delta_{N} H_{i}^{n+1}}\left[N_{i}^{n}+\Delta t N_{i}^{n}\left(1-\theta K_{i}^{n}-N_{i}^{n}\right)\right]
$$

for each space point $i$.

In order to write the term characterizing the dispersion of the neoplastic tissue into the healthy tissue we make use of the nonstandard finite difference scheme [24], that is,

$$
\begin{aligned}
\left.\nabla(D(N) \nabla K)\right|_{x_{i}}=\frac{1}{2(\Delta x)^{2}} \sum_{k \in N_{i}}( & \left.D\left(N_{k}^{n+1}\right)+D\left(N_{i}^{n+1}\right)\right) \\
& \times\left(K_{k}^{n+1}-K_{i}^{n+1}\right),
\end{aligned}
$$

where $D(N)=\delta_{k}(1-N)$ and $N_{i} \subset I$ is the index set pointing at the direct neighbours of $x_{i}$ on the grid. Thus $N_{i}$ has two elements for the one-dimensional case and four elements for the two-dimensional case and the matrix-vector form of the scheme reads

$$
\mathbf{A}_{\mathbf{K}} \mathbf{K}^{n+1}=\mathbf{K}^{n}+\vartheta_{\mathbf{K}}^{\tilde{n}}
$$

where $\mathbf{A}_{\mathbf{K}}$ is the $(m+1) \times(m+1)$ tridiagonal matrix or the block tridiagonal matrix of size $(m+1)^{2} \times(m+1)^{2}$ for the oneand two-dimensional cases, respectively, coming from the nonstandard finite difference discretization, $\vartheta_{\mathrm{K}}^{\tilde{n}}$ is the vector coming from the proliferation term with the entries $\rho_{k} K_{i}^{n}(1-$ $K_{i}^{n}-N_{i}^{n+1}$ ), and $\mathbf{K}^{n+1}$ and $\mathbf{K}^{n}$ are the vectors containing the $K$ values at the discretized points for the time levels $n+1$ and $n$, respectively.

Throughout our simulations we use the biological parameter values from Table 1 which is reproduced from [24].

According to a linear stability analysis performed in [24], the parameter $\delta_{N}$ plays a crucial role in characterizing the aggressivity of the tumor. There, $\delta_{N}=1$ was shown to be the crossover value; for $\delta_{N}<1$ the tumor is less aggressive, whereas for $\delta_{N}>1$ it becomes highly aggressive. This will be an important factor in our computations, too.

4.1. The One-Dimensional Case. We consider the space interval $[0,1]$ and choose for the time and space increments $\Delta t=$ 0.1 and $\Delta x=0.01$, respectively.

In Figures 1 and 2 we present the simulations with $\delta_{N}=$ 50 (an aggressive tumor) and $\delta_{N}=0.5$ (a less-aggressive one), respectively. The rest of parameters are taken from Table 1. As time progresses, the difference between these two cases is more visible. In the aggressive case at later times the cancer cells invade a larger region and destroy the healthy tissue much more than in the case of a less-aggressive tumor (Figure 2).

In the next set of graphs we consider the negative effect of the aggressivity parameter $\delta_{N}$ on the normal cell density. We know from the nondimensionalization addressed in Section 2 that the (nondimensionalized) parameter $\delta_{N}$ is proportional to the death rate $d_{N}$ and inversely proportional to the proton reabsorption rate $d_{H}$.

Figure 3(a) shows the evolution of the normal cell density with respect to $\delta_{N}$ for the times up to $t=40$ at a fixed space point $x=0.4$. One can notice that a more aggressive tumor (larger $\delta_{N}$ or equivalently larger $d_{N}$ ) leads to a faster decay in the density of the normal cells, as expected. On the other hand, Figure 3(a) also supports the intuitive fact that in an organism which can poorly buffer the issuing excessive protons (smaller $d_{H}$ and larger $\delta_{N}$, resp.) the $\mathrm{pH}$ value will decay faster as well, thus triggering the decay of normal cell density, which in such an acid environment can no longer be sustained at a physiologically convenient level.

Figure 3(b) illustrates the normal cell density at $t=10$ depending on the concentration of $\mathrm{H}^{+}$protons for several different values of $\delta_{N}$. In an organism whose normal cells are more sensitive to $\mathrm{pH}$ variations (larger $d_{N}$ ) the density of these cells will decay faster for the same concentration of $\mathrm{H}^{+}$protons. It can also be seen that a smaller reabsorption 
rate of excessive protons leads to a faster decay of normal cell density.

4.2. The Two-Dimensional Case. We perform $2 \mathrm{D}$ simulations in the unit square $[0,1] \times[0,1]$ using $\delta_{H}=70$ and still with the parameter values in Table 1 . Analogously to our computations in $1 \mathrm{D}$ we consider two different cases: $\delta_{N}=$ 12.5 (an aggressive tumor) and $\delta_{N}=0.5<1$ (a lessaggressive one). We use $\Delta t=0.01$ as the time increment and for the spatial discretization we take 11 nodes on each axis, leading to 121 nodes in the computational domain.

The evolution of cancer cells is plotted in Figure 4. At an earlier time (e.g., $t=1$, see Figures $4(\mathrm{a})$ and $4(\mathrm{~d})$ ) the difference between the less- and the higher-aggressive tumors is not relevant. However, as time progresses the difference starts to be visible (e.g., at $t=10$, see Figures 4(b) and 4(e)), whereas by $t=50$ the more-aggressive tumor (Figure 4(c)) invaded almost the whole domain and the less-aggressive one was not able to penetrate that far.

Also observe that the proton concentration varies proportionally to the tumor cell density and is inversely proportional to the normal cell density (Figures 5 and 6, resp.). Moreover, the healthy tissue is completely destroyed in the case of an aggressive tumor (Figure 6(c)) by $t=50$.

\section{Acknowledgments}

C. Surulescu acknowledges the support of the BadenWürttemberg Foundation. G. Meral acknowledges the support of LLP Erasmus Staff Mobility Programme during her visit to the University of Kaiserslautern.

\section{References}

[1] A. Ashkenazi and V. M. Dixit, "Death receptors: signaling and modulation," Science, vol. 281, no. 5381, pp. 1305-1308, 1998.

[2] H. Yamaguchi, F. Pixley, and J. Condeelis, "Invadopodia and podosomes in tumor invasion," European Journal of Cell Biology, vol. 85, no. 3-4, pp. 213-218, 2006.

[3] J. Kelkel and C. Surulescu, "On some models for cancer cell migration through tissue networks," Mathematical Biosciences and Engineering, vol. 8, no. 2, pp. 575-589, 2011.

[4] J. Kelkel and C. Surulescu, "A multiscale approach to cell migration in tissue networks," Mathematical Models and Methods in Applied Sciences, vol. 22, no. 3, Article ID 1150017, 25 pages, 2012.

[5] T. Hillen, " $\mathrm{M}^{5}$ mesoscopic and macroscopic models for mesenchymal motion," Journal of Mathematical Biology, vol. 53, no. 4, pp. 585-616, 2006.

[6] A. Chauviere, T. Hillen, and L. Preziosi, "Modeling cell movement in anisotropic and heterogeneous network tissues," Networks and Heterogeneous Media, vol. 2, no. 2, pp. 333-357, 2007.

[7] A. R. A. Anderson, M. A. J. Chaplain, E. L. Newman, R. J. C. Steele, and A. M. Thompson, "Mathematical modeling of tumor invasion and metastasis," Journal of Theoretical Medicine, vol. 2, pp. 129-154, 2000.

[8] R. A. Gatenby and E. T. Gawlinski, "A reaction-diffusion model of cancer invasion," Cancer Research, vol. 56, no. 24, pp. 57455753, 1996.
[9] R. A. Gatenby and R. J. Gillies, "Glycolysis in cancer: a potential target for therapy," International Journal of Biochemistry and Cell Biology, vol. 39, no. 7-8, pp. 1358-1366, 2007.

[10] D. Hanahan and R. A. Weinberg, "Hallmarks of cancer: the next generation,” Cell, vol. 144, no. 5, pp. 646-674, 2011.

[11] H. Izumi, T. Torigoe, H. Ishiguchi et al., "Cellular $\mathrm{pH}$ regulators: potentially promising molecular targets for cancer chemotherapy," Cancer Treatment Reviews, vol. 29, no. 6, pp. 541-549, 2003.

[12] O. R. Abakarova, "The metastatic potential of tumors depends on the $\mathrm{pH}$ of host tissues," Bulletin of Experimental Biology and Medicine, vol. 120, no. 6, pp. 1227-1229, 1995.

[13] R. Martínez-Zaguilán, E. A. Seftor, R. E. B. Seftor, Y. W. Chu, R. J. Gillies, and M. J. C. Hendrix, "Acidic $\mathrm{pH}$ enhances the invasive behavior of human melanoma cells," Clinical and Experimental Metastasis, vol. 14, no. 2, pp. 176-186, 1996.

[14] R. A. Gatenby and E. T. Gawlinski, "The glycolytic phenotype in carcinogenesis and tumor invasion: insights through mathematical models," Cancer Research, vol. 63, no. 14, pp. 3847-3854, 2003.

[15] A. Fasano, M. A. Herrero, and M. R. Rodrigo, "Slow and fast invasion waves in a model of acid-mediated tumour growth," Mathematical Biosciences, vol. 220, no. 1, pp. 45-56, 2009.

[16] K. Smallbone, D. J. Gavaghan, R. A. Gatenby, and P. K. Maini, "The role of acidity in solid tumour growth and invasion," Journal of Theoretical Biology, vol. 235, no. 4, pp. 476-484, 2005.

[17] L. Bianchini and A. Fasano, "A model combining acid-mediated tumour invasion and nutrient dynamics," Nonlinear Analysis: Real World Applications, vol. 10, no. 4, pp. 1955-1975, 2009.

[18] J. Kelkel and C. Surulescu, "A weak solution approach to a reaction-diffusion system modeling pattern formation on seashells," Mathematical Methods in the Applied Sciences, vol. 32, no. 17, pp. 2267-2286, 2009.

[19] J. Kelkel and C. Surulescu, "On a stochastic reaction-diffusion system modeling pattern formation on seashells," Journal of Mathematical Biology, vol. 60, no. 6, pp. 765-796, 2010.

[20] A. S. Silva, J. A. Yunes, R. J. Gillies, and R. A. Gatenby, “The potential role of systemic buffers in reducing intratumoral extracellular $\mathrm{pH}$ and acid-mediated invasion," Cancer Research, vol. 69, no. 6, pp. 2677-2684, 2009.

[21] I. F. Tannock and D. Rotin, "Acid pH in tumors and its potential for therpeutic exploitation," Cancer Research, vol. 49, no. 16, pp. 4373-4384, 1989.

[22] L. C. Evans, Partial Differential Equations, vol. 19, American Mathematical Society, Providence, RI, USA, 1998.

[23] A. D. Polyanin, Handbook of Linear Partial Differential Equations for Engineers and Scientists, Chapman \& Hall, New York, NY, USA, 2002.

[24] H. J. Eberl and L. Demaret, "A finite difference scheme for a degenerated diffusion equation arising in microbial ecology," Electronic Journal of Differential Equations, vol. 15, pp. 77-95, 2007. 


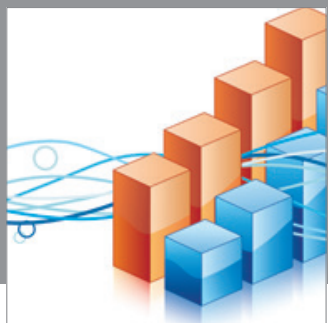

Advances in

Operations Research

mansans

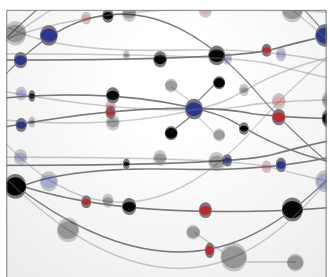

The Scientific World Journal
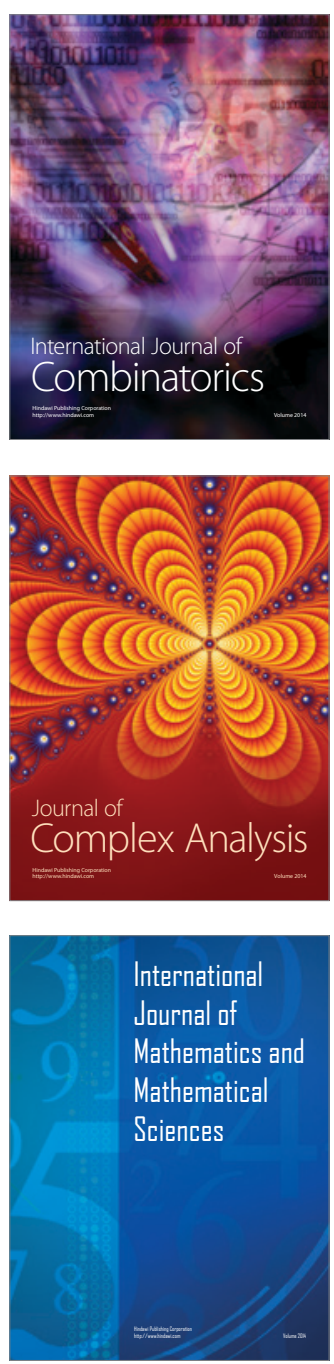
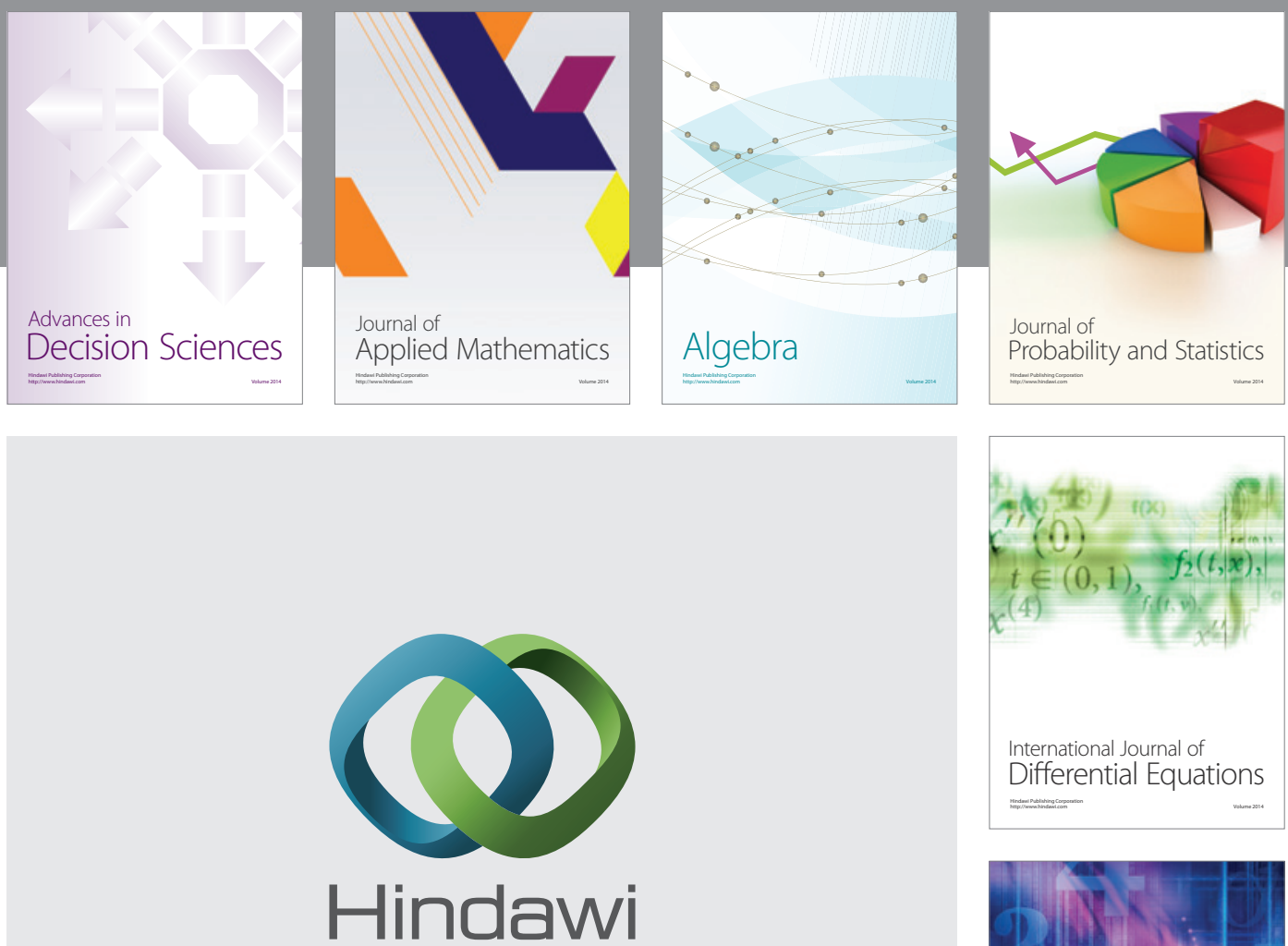

Submit your manuscripts at http://www.hindawi.com
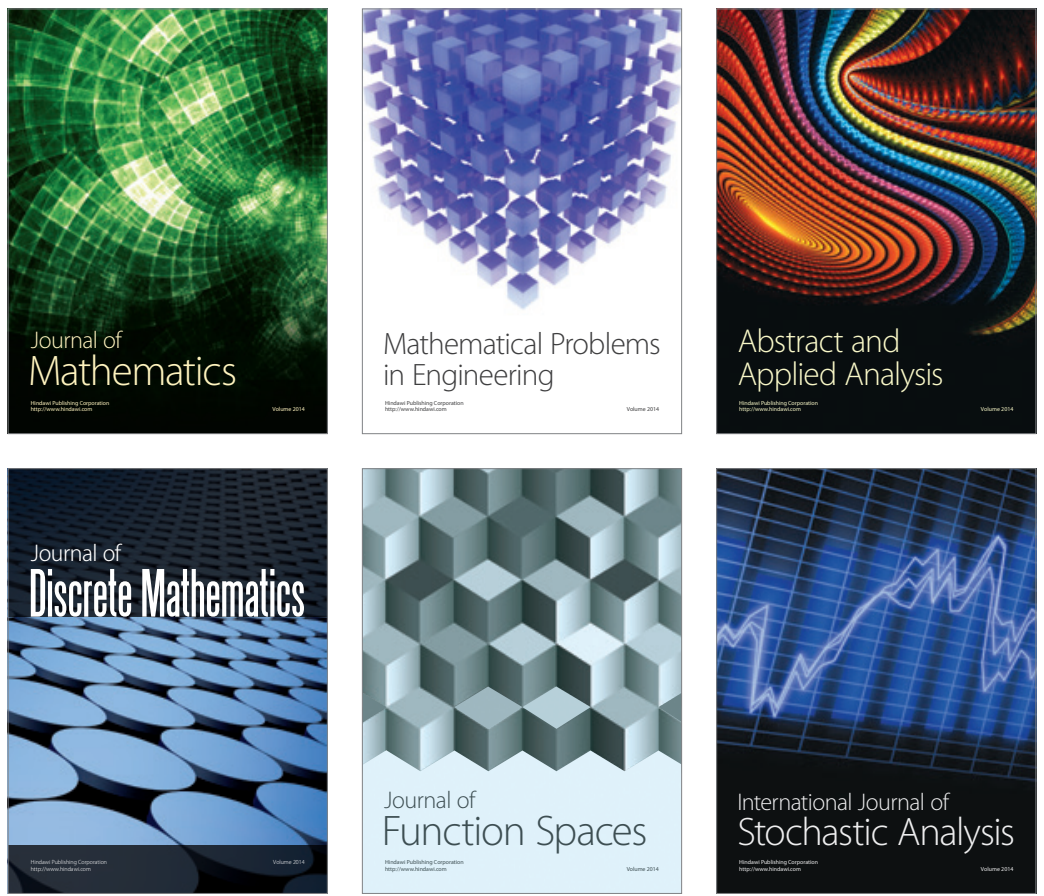

Journal of

Function Spaces

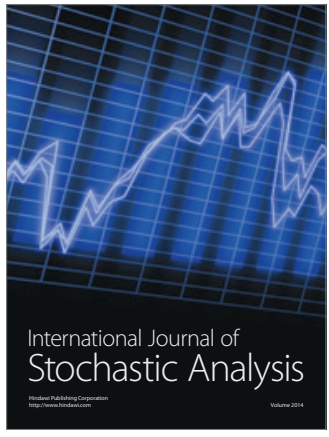

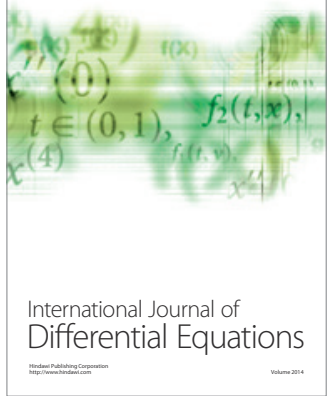
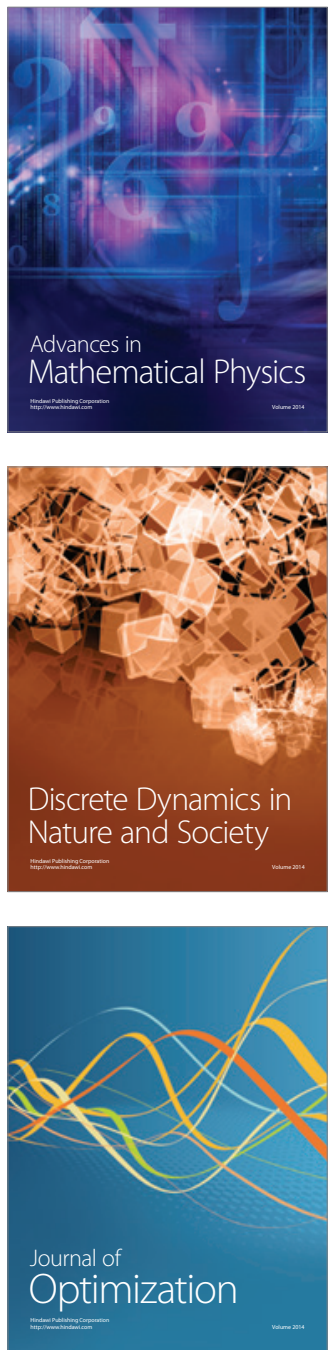